\title{
Stereo-motion cooperation and the use of motion disparity in the visual perception of 3-D structure
}

\author{
VALERIE CORNILLEAU-PERES and JACQUES DROULEZ \\ Laboratoire de Physiologie Neurosensorielle, CNRS, Paris, France
}

\begin{abstract}
When an observer views a moving scene binocularly, both motion parallax and binocular disparity provide depth information. In Experiments 1A-1C, we measured sensitivity to surface curvature when these depth cues were available either individually or simultaneously. When the depth cues yielded comparable sensitivity to surface curvature, we found that curvature detection was easier with the cues present simultaneously, rather than individually. For 2 of the 6 subjects, this effect was stronger when the component of frontal translation of the surface was vertical, rather than horizontal. No such anisotropy was found for the 4 other subjects. If a moving object is observed binocularly, the patterns of optic flow are different on the left and right retinae. We have suggested elsewhere (Cornilleau-Pérès \& Droulez, in press) that this motion disparity might be used as a visual cue for the perception of a 3-D structure. Our model consisted in deriving binocular disparity from the left and right distributions of vertical velocities, rather than from luminous intensities, as has been done in classical studies on stereoscopic vision. The model led to some predictions concerning the detection of surface curvature from motion disparity in the presence or absence of intensity-based disparity (classically termed binocular disparity). In a second set of experiments, we attempted to test these predictions, and we failed to validate our theoretical scheme from a physiological point of view.
\end{abstract}

The architecture of the visual receptors, as well as the retinotopic organization of the first neuronal networks in the visual pathway, indicates that primary visual information is essentially bidimensional. Among the sensory cues used by the central nervous system to deduce the three-dimensional (3-D) structure of a visual scene from retinal images, binocular disparity and motion parallax have been studied most, both experimentally and theoretically. Although other depth cues such as accommodation, convergence, or spatial and temporal variations of texture can also provide depth information to an observer (Foley, 1978; Stevens \& Brookes, 1988), motion parallax and stereopsis are likely to play a major role in depth perception. Recently, several authors have described responses to conflicting stereoscopic and motion information (Braunstein, Andersen, Rouse, \& Tittle, 1986; Rogers \& Collett, 1989). In the present study, we address the problem of the interaction and cooperation of motion parallax and stereopsis when they both participate coherently in the visual perception of 3-D structure.

Both of these depth cues provide a very accurate perception of relative depth in some spatial and temporal conditions (Rogers \& Graham, 1985), and both are coded in the primary steps of cortical visual processing. They can also be used in the absence of monocular object identification; binocular disparity is isolated in the random-

This work was supported by the company Essilor (Convention CIFRE No. 85/224) and by the program COGNISCIENCES of the CNRS. We thank Brian Rogers for helpful discussion. Correspondence should be sent to V. Cornilleau-Pérès, Laboratoire de Physiologie Neurosensorielle, 15 rue de l'École de Médecine, 75270 Paris Cedex 06, France. dot stereograms designed by Julesz (1971), and motion parallax remains the only depth cue (although this is still discussed, as will be explained below) in the random-dot kinematograms used in the numerous studies of the kinetic depth effect that have been done since the original experiments of Green's (1959).

The mathematical problems of reconstructing the third dimension from motion or from stereopsis are very similar. In both cases, 3-D geometrical parameters have to be deduced from several views of the same visual scene. A first step consists in the pairing of image points that are images of the same 3-D point across different views. In the motion parallax case, the correspondence is established for successive images and yields a velocity or displacement field, whereas in the stereopsis case, the two views are compared simultaneously and the correspondence is coded as a disparity field. The theoretical approach to structure from motion (Droulez \& Cornilleau-Pérès, 1990, Longuet-Higgins \& Prazdny, 1980; Waxman \& Ullman, 1985 ) is often based on the assumption that, at a given step in the process, the set of velocities (or the velocity field) of each image point on the retina is coded in the visual pathway and constitutes an input for the 3-D computation. It should be pointed out that many theoretical studies have shown that to deduce the velocity field from patterns of fluctuating luminous intensities is not a straightforward operation (Hildreth, 1984; Horn \& Schunck, 1981; Verri \& Poggio, 1986). Therefore, even if there is good physiological evidence for the presence of velocity detectors in the visual pathway (for a review see, e.g., Nakayama, 1985), the existence of a separate process of velocity computation feeding a 3-D structure-from-motion 
step remains a hypothesis. Similarly, detectors of binocular disparity have been found in the first cortical steps of visual processing, but it remains to be understood how depth is encoded by the brain from the activity of these neurons.

Finally, motion parallax and stereopsis display some kinds of interactions (Nawrot \& Blake, 1991; Rogers \& Collett, 1989), in the sense that stereopsis can modify the perceived 3-D movement of an object specified by motion parallax.

When an observer views a moving object binocularly, depth information is provided by motion parallax in each monocular view, as well as by the binocular disparity between relative positions of image points on the left and right retinae (we call this cue position disparity). At a given instant, those two images also present a difference of velocity; for example, if a point moves along the sagittal axis of the subject, its projected velocities on the left and right retinae are opposite in direction (Beverley \& $\mathbf{R e}$ gan, 1973).

The studies of dynamic stereopsis discussed so far are related to horizontal disparity changes. Given a point $M$ moving in space in relation to a stationary fixation point $F$, if $a_{1}$ and $a_{2}$ are the horizontal angular extents between $M$ and $F$, as seen from the left and right eyes, respectively, the instantaneous horizontal disparity is $d=$ $a_{1}-a_{2}$. Its temporal variation relative to time $t$ is $\dot{d}$, a quantity often termed the rate of change of disparity. It is also equal to the difference $u_{1}-u_{2}$ between the left and right horizontal image velocities of $M$, which we call motion disparity. The rate of change of disparity and motion disparity therefore correspond to the same mathematical variable, which we shall call horizontal dynamic disparity. However, the use of one or the other term bears implications regarding the processes underlying the computation of this quantity-in particular, regarding their interdependencies with the computation of image velocities (the rate of change of disparity can be computed independently from image velocities). Up to now, the studies related to horizontal dynamic disparities have suggested the following:

1. This variable is coded in the visual system as a cue for the perception of motion in depth. In the cat, cells that are specifically sensitive to this variable have been found in area 18 (Cynader \& Regan, 1978, 1982; Orban, Spileers, Gulyas, \& Bishop, 1986; Pettigrew, 1973) and in the Clare-Bishop area (Toyama \& Kozasa, 1982; Toyama, Komatsu, Kasai, Fujii, \& Umetani, 1985). With respect to the monkey, this is still debated (Maunsell \& van Essen, 1983; Poggio \& Talbot, 1981; Zeki, 1974). As for humans, Beverley and Regan (1973) obtained psychophysical evidence for the existence of a separate visual "channel" responding selectively to changing horizontal disparities, which was supported by subsequent studies (Regan \& Beverley, 1973; Richards, 1977; Richards \& Regan, 1973).

2. The horizontal dynamic disparities of an object defined solely by kinetic contours can reveal motion in depth. Lee (1971) used random-dot stereokinematograms representing a vertical strip that moved in front of a static background with both frontal and in-depth translations (each point of the strip depicted the same elliptical trajectory in a horizontal plane). The kinematograms viewed by the left and right eyes were calculated from different dot distributions. Each yielded the perception of a vertical strip oscillating in a frontal plane when viewed monocularly. However, when viewed stereoscopically, the two films induced the perception of the 3-D motion of the strip, although each stereo pair of images was devoid of any depth information.

In the present paper, we describe a psychophysical investigation of dynamic stereopsis based on a theoretical approach that involves mainly vertical image velocities. In a previous paper (Cornilleau-Pérès \& Droulez, in press), we have shown that vertical image velocities could in principle replace image luminous intensities for the calculation of binocular disparities. Indeed the implicit assumption that "corresponding points should have the same value of a function $I$ derived from the luminous intensity" is simply replaced here by the assumption that "corresponding points should have the same value of a function $f$ derived from vertical image velocities." In computer simulations, we found that for many 3-D movements of rigid objects (although our scheme does not require any rigidity assumption), to establish a binocular correspondence between the left and right distributions of vertical velocities was possible and that the subsequent recovery of object structure presented robustness to noise. These simulations showed that the quality of depth recovery depended on the type of 3-D movement, and in particular on the direction of the frontal translation involved, in comparison with the direction of the interocular segment.

To assume that the visual system uses vertical image velocities to process binocular depth information leads us to two predictions: (1) When both stereopsis and motion parallax are available as depth cues, there should be an effect of cooperation, in the sense proposed by Bülthoff and Mallot (1987) (two depth cues have cooperative interactions if there is greater facilitation than would be predicted by probability summation). (2) This effect of cooperation should vary with the type of 3-D movement involved, and in particular with the direction of the frontal translation. These predictions were tested in a first series of experiments involving the detection of surface curvature from stereokinetic images.

In a second set of experiments, we tested whether the visual system could process a velocity-based correspondence from images that were binocularly correlated in velocity but not in luminance. As in Lee's (1971) experiment, we presented to each eye a sequence of images representing a moving surface defined by a set of points. Both sequences were calculated as viewed from each eye, but the set of points defining the surface was different, depending on the destination of the sequence (left or right 
eye). In the control stimuli, we also suppressed motion disparity by calculating both sequences as viewed from the left eye.

In a recent series of papers, researchers have intensively discussed the validity of experimental procedures used to study the kinetic depth effect. In light of this discussion, we will first analyze our own procedure in detail. Then we will summarize our theoretical scheme of motion disparity processing, and its psychophysical implications, and we will describe a quantitative analysis of stereomotion cooperation that will serve the interpretation of our experiments. Finally, we will present the two sets of experiments.

\section{THE PARADIGM OF DETECTION OF SURFACE CURVATURE FROM MOTION OR STEREO}

\section{Studying the Kinetic Depth Effect Experimentally}

Recently three consecutive papers (Braunstein \& Todd, 1990; Sperling, Dosher, \& Landy, 1990; Sperling, Landy, Dosher, \& Perkins, 1989) have discussed different points concerning the experimental study of the kinetic depth effect. All these authors agree that the exact field of application of each experiment should be specified (e.g., "structure from motion," or "structure from motion and texture"). In particular, in any experiment strictly related to the kinetic depth effect, it should be carefully ensured that the subjects can base their responses only on the perceived depth and not on (1) artifacts (properties of the stimuli incidentally related to the 3-D structure of the stimuli) or (2) alternative computations (cues that covary with surface structure in natural conditions). The authors also emphasize the importance of the subjects' verbal reports, which can strengthen the validity of a paradigm regarding the perception of depth. Indeed if the brain effectively codes differential velocities and uses them as input for depth perception, it is impossible to distinguish objectively between experiments related to the perception of relative velocities and to the kinetic depth effect. The only arguments that allow one to differentiate between these two types of experiments concern the subjects' verbal reports and the type of verbal instructions given to the subjects prior to the experiment.

The question of using feedback and experienced subjects rather than no feedback and naive subjects has been strongly debated. We believe that there is no conclusive argument for choosing between the two solutions, as their coexistence in the literature on psychophysics in general illustrates. Rather, we would formulate questions, whenever possible, that can be answered through the comparison of two experimental conditions performed by the same subject on the same day. Of course as a complement it is necessary to verify that any differences in responses between the two conditions cannot be due to an artifact or alternative computation.

\section{The Paradigm of Curvature Detection}

The present investigation was based on a paradigm that we have already used to study the visual perception of cylinder curvature (Cornilleau-Pérès \& Droulez, 1989). A patch of dotted surface is viewed through a circular aperture (drawn on the screen). In the first image (hereafter designated as the median image), the surface lies normal to the sagittal axis of the subject, and a set of dots is randomly spread over the surface, with a 2-D uniform density (i.e., the density is uniform on the screen). In the motion conditions, the 2-D coordinates of the dots are calculated for different successive positions of the surface. The set of images thus obtained is displayed to the subject, simulating the real view of a moving surface. In the stereo conditions, pairs of stereoscopic images of the surface are calculated and fused by the subjects through displacing prisms.

In the present study, the surfaces were spherical or planar. The radius of the sphere was constant during each experimental session, and the subject had to indicate whether he/she perceived the surface as spherical or planar. All but one of the subjects (one of the authors) were naive; none received feedback. A preliminary training session allowed us to check that at supraliminary levels they were able to describe the shape of different types of surfaces verbally.

\section{Possible Artifacts}

In the motion condition, the following variables were considered as possible artifacts of our paradigm: (1) the 2 -D velocity magnitude of the dots; (2) the 2-D acceleration magnitude of the dots; (3) the spatial arrangement of dots in the extreme image (i.e., the image displaying the surface at its maximum tilt angle); and (4) the total number of dots in the extreme image.

In Experiment 1C, we ensured that the subjects could not base their responses on these variables. This we achieved by comparing the performance obtained when these variables covaried or did not covary with surface curvature. Of course there might exist other artifacts, and there is probably no way to eliminate all of them. However, it is very likely that such other artifacts are considerably weakened when the correlation between surface curvature and the values of the variables listed above is suppressed.

\section{Alternative Computations}

The elimination of the static spatial arrangement of the dots as an artifact does not rule out the possibility that subjects could base their responses on the dynamic changes of this parameter. Indeed spatial variations of the velocity field necessarily imply local changes in the distribution of the dots. Our paradigm (like many others in the literature) rigorously addresses the study of the perception of structure from motion parallax and temporal variations in dot density occurring in different parts of 
the image. However, our 1989 study suggests that the latter variable probably plays a secondary role. The anisotropy concerning the direction of motion relative to the direction of the cylinder axis could be explained by a coding of a second spatial derivative of the velocity field (the spin variation), whereas the operator quantifying the local increase of dot density predicted an anisotropy that was opposite to our observations. Finally, the adequacy of our results (1989) with respect to the prediction of the spin variation theory suggests that our paradigm is a pure kinetic depth effect paradigm in the sense that spatial variations of the velocity field constitute the cue for the perception of surface curvature.

\section{COMPUTING 3-D STRUCTURE FROM MOTION DISPARITY}

\section{The Theoretical Scheme}

Several theoretical studies have addressed the problem of stereo-motion cooperation in the processing of 3-D structure and motion. In most of them, it has been supposed that the stereo correspondence has been established for each image pair, and that the optic flow is available for each monocular image sequence (Jenkin, 1984; Mitiche, 1984, 1988; Richards, 1983). Waxman and Duncan (1985) have shown that motion and stereopsis can cooperate earlier-namely, while the stereo correspondence between pairs of images is being established. However, Waxman and Duncan's theoretical approach was limited to the study of rigid surfaces of low curvature, and they did not test its physiological and (in terms of robustness to noise) computational validity.

In previous papers (Cornilleau-Pérès \& Droulez, 1990, in press), we have shown that, in many cases, it is possible to use the binocular optical flows directly for stereo matching. Briefly, consider the two retinae as approximated by two hemispheres of centers $O_{1}$ and $O_{2}$, the nodal points of the left and right eyes, respectively (Figure 1A). (This is by no means a restrictive hypothesis, since metrical transformations can map the actual retinae onto such hemispheres.) The epipolar constraint states that, given a point $M_{1}$ on the left retina, its corresponding point $M_{2}$ on the right retina necessarily lies in the same plane as $M_{1}, O_{1}$, and $O_{2}$. In other words, if the geometry of the viewing system is known, which we state as a hypothesis, the problem of stereo matching consists in the pairing of points lying on the corresponding epipolar lines $E_{1}$ and $E_{2}$, located at the intersection of the retinae with a plane containing $\mathrm{O}_{1}$ and $\mathrm{O}_{2}$.

Given a point $M_{1}$ on the left retina, there exists a unique plane $P$ containing $M_{1}, O_{1}$, and $O_{2}$. The vector normal to this plane is $\mathbf{j}$ (in current situations, $P$ is roughly horizontal, while $\mathrm{j}$ is roughly vertical), and $\theta_{1}$ and $\theta_{2}$ are the eccentricities of points $M_{1}$ and $M_{2}$ of the left and right retinae, defined in plane $P$ as illustrated in Figure $1 \mathrm{~A}$.

If $V_{1}$ and $V_{2}$ represent the velocity vectors of $M_{1}$ and $M_{2}$, we call $v_{1}$ and $v_{2}$ their components along the direction $\mathrm{j}$, which is tangent to both retinae in $M_{1}$ and $M_{2}$ (Fig-

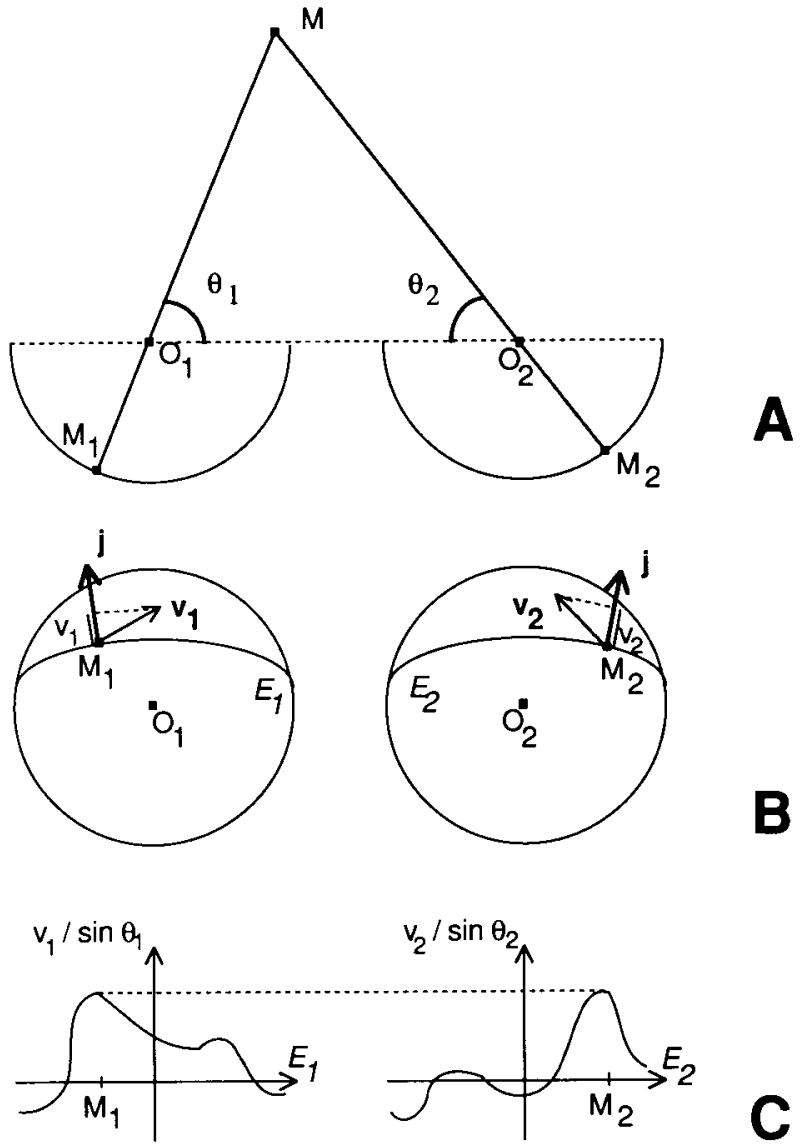

Figure 1. The theoretical scheme. (A) $O_{1}$ and $O_{2}$ are the nodal points of the left and right eyes. The left and right retinae are approximated by the hemispheres centered on $O_{1}$ and $O_{2} . M$ is an object point, $M_{1}$ and $M_{2}$ its left and right images, which have $\theta_{1}$ and $\theta_{2}$ as retinal eccentricities in the plane $\left(O_{1} O_{2} M\right)$. (B) $E_{1}$ and $E_{2}$ are the two corresponding epipolar lines included in the left and right retinae and passing through the points $M_{1}$ and $M_{2}$, respectively. $V_{1}$ and $V_{2}$ are the retinal velocities of the points $M_{1}$ and $M_{2} . j$ is the vector normal to the plane $\left(O_{1} O_{2} M\right)$ and tangent to the retinae in $M_{1}$ and $M_{2} \cdot v_{1}$ and $v_{2}$ are the components of $v_{1}$ and $v_{2}$ along $j$. (C) The function $f$ (see text for definition) can be calculated along $E_{1}$ and $E_{2}$. Corresponding points of these epipolar lines necessarily present the same value of function $f$.

ure 1B). If there exists an object point $M$ projecting in $M_{1}$ and $M_{2}$, its 3-D velocity vector $\mathrm{U}$ verifies that

$$
v_{1}=\langle\mathbf{U}, \mathbf{j}\rangle / O_{1} M
$$

and

$$
v_{2}=\langle\mathbf{U}, \mathbf{j}\rangle / O_{2} M,
$$

where $\langle$,$\rangle is the scalar product, and O_{1} M$, the distance between $O_{1}$ and $M$. A simple trigonometrical calculation shows that

$$
\sin \theta_{1} \cdot O_{1} M=\sin \theta_{2} \cdot O_{2} M,
$$

and the elimination of $\langle\mathbf{U}, \mathbf{j}\rangle$ from the preceding equations yields 


$$
v_{1} / \sin \theta_{1}=v_{2} / \sin \theta_{2} .
$$

A possible process of stereo correspondence thus consists in the pairing of points that lie on corresponding epipolar lines and that present the same value of the function

$$
f(\theta)=v / \sin \theta .
$$

(We drop the indices 1 and 2 with straightforward notations.)

This process could be named stereo from vertical motion disparity, as opposed to the classical stereo from luminous intensity, since the function $f$ depends mainly on the vertical velocity component. Note that it does not require any hypothesis concerning the object under analysis and its 3-D motion, and that its performance depends on the variations of the function $f$ along an epipolar line. These variations are not always sufficient for the pairing of image points. We have discussed the possible ambiguities in the case of a rigid object. Our conclusions can be summarized as follows. Decomposing the 3-D motion into a rotation around the point $O$, the middle of the segment $\left(O_{1} O_{2}\right)$, and a translation, we reached the conclusion that, except for movements composed only of a translation along $\left(O_{1} O_{2}\right)$ and a rotation around $\left(O_{1} O_{2}\right)$, the process of stereo correspondence based on the function $f$ is theoretically possible. In addition, the highest robustness to noise is obtained when the surface presents steep variations in depth in the direction $\left(\mathrm{O}_{1} \mathrm{O}_{2}\right)$ and when the image is stabilized. The former result reflects the need for high variations of function $f$ along epipolar lines, similar to the need for variations of luminous intensity in the horizontal direction for intensity-based stereopsis (recall that, for all movements involving a frontal translation, image velocity varies with depth). The latter constraint is acceptable for human vision, because for a wide range of global velocities image stabilization can be achieved by means of eye movements.

\section{Psychophysical Implications}

Assuming that motion disparity can be used by the visual system as a depth cue according to a process of the type described above, we have developed quantitative predictions concerning the detection of surface curvature by a human observer. These predictions are founded on results of simulations (Cornilleau-Pérès \& Droulez, in press) that we summarize here. The inputs were synthetic binocular velocity fields of moving objects, from which we calculated the theoretical value of function $f$ on a $40 \times 40$ pixel grid. A simple algorithm of stereoscopic matching was then applied; the correspondent to each left pixel was found in the right image by linear interpolation of function $f$ along the epipolar line. The objects under analysis were rigid surfaces for which function $f$ did not present large discontinuities, and, for each 3-D movement where function $f$ varied along the epipolar lines, the object structure was therefore perfectly reconstructed. Since exact velocity fields are very unlikely to be computed by machine or biological vision systems, the in- teresting point was to test the robustness of this algorithm to noise. Therefore, we used synthetic velocity fields that were perturbed by two Gaussian noises: (1) a proportional noise of standard deviation $s_{1}$, which was proportional to the absolute value of the vertical velocity (3\% of this value) in each pixel; and (2) a background noise of standard deviation $s_{2}$, which was constant over the image (2.5\% of the maximum value of the vertical velocity component over the image). If $r_{1}$ and $r_{2}$ are two random Gaussian variables of standard deviations $s_{1}$ and $s_{2}$, the vertical component of the velocity that serves as an input to the algorithm is $v+r_{1}+r_{2}$, where $v$ is the theoretical value of that component.

Our velocity-correspondence scheme is able to recover depth only when the input function $f$ varies along epipolar lines. Therefore, a step in selection of the image pixels whereby depth could in principle be calculated was performed prior to the application of the algorithm, according to two criteria: The relative variations of function $f$ along an epipolar had to be higher than $1 \%$ between neighboring pixels; and the absolute value of function $f$ had to be higher than four times the background noise.

The percentage of these high-confidence points and the relative error performed on the reconstructed depth are two measurements of the algorithm's performance. These quantities were computed for different objects, including planar and spherical surfaces (with a radius of $1 \mathrm{~m}$ in the latter case), with orientation normal to the sagittal axis $(O K)$ or inclined by $12.45^{\circ}$ in the vertical or horizontal direction (this is the extreme position reached by these surfaces in the psychophysical experiments).

The surface motion was decomposed in a rotation around point $O$ (midway between $O_{1}$ and $O_{2}$ ), a frontal translation (normal to the sagittal axis), and a translation in depth (parallel to the sagittal axis). We used two types of motion.

Motion $R$ was a rotation around a frontal axis passing through point $K$, the intersection of the surface and the sagittal axis. The rotation axis could be either vertical or horizontal (Figures 2A-2B), and the motion was then called $R_{\mathrm{h}}$ or $R_{\mathrm{v}}$, respectively (this is due to the fact that the component of frontal translation was orthogonal to the rotation axis).

Motion $O$ was a rotation around the sagittal axis (Figure 2C).

Both motions yield a zero velocity in the centers of the images viewed by the left and right eyes, which ensures a maximum robustness to the component of proportional noise of the theoretical scheme (the perturbation on the spatial variations of $f$ due to this noise increases with the global level of function $f$ ). As far as monocular motion parallax is concerned, motion $R$ can reveal the 3-D structure of an object to the observer, since it presents a component of frontal translation (Cornilleau-Pérès \& Droulez, 1989). Motion $O$, on the other hand, is nearly a pure rotation around the nodal points of the left and right eyes and is therefore likely to yield no perception of 3-D structure through motion parallax (a pure rotation around an eye does not provide any information of structure from motion). 


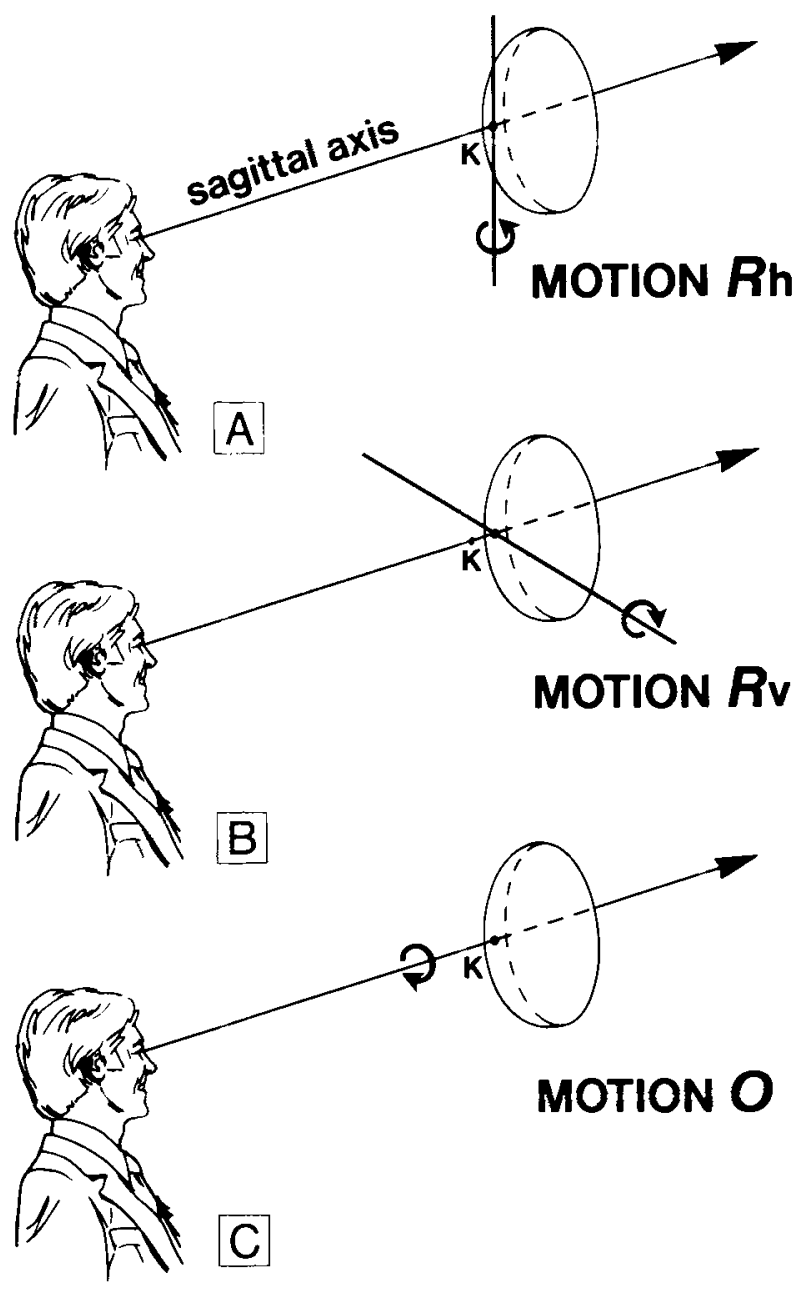

Figure 2. The 3-D motions used in the experiments. Point $K$ is located at the intersection of the sagittal axis and the surface. (A) Motion $R$ is a rotation around a frontal axis passing through $K$. This axis can be vertical, and the component of frontal translation is then horizontal (motion $R_{\mathrm{h}}$ ). (B) When the rotation axis is horizontal, the component of frontal translation is vertical (motion $R_{v}$ ). (C) Motion $O$ is a rotation around the sagittal axis.
The image subtended $8^{\circ}$ of visual angle, as in the psychophysical experiments. Note that the size of 40 pixels is not to be compared with the resolution of our experimental setup, one reason being that, in our simulations, we used theoretical velocity fields (calculated with high precision) to which we added noise, whereas the velocity input to the visual system depends on both the resolution of our display and the performance on velocity computation by the visual system. The goal of our simulations was not to make these velocity inputs similar, but rather to compare the performance of our scheme for different types of 3-D movements.

The results are presented in Table 1 . For the plane normal to the sagittal axis and motion $R$, the results have not been included, since the reconstruction of depth from motion disparity is impossible in this case. For motion $R$, the extreme position corresponds to the tilt of $12.45^{\circ}$, which is reached by the surface during the corresponding movement (horizontal tilt for motion $R_{\mathrm{h}}$, vertical tilt for motion $R_{\mathrm{v}}$ ). The results in Table 1 show that the algorithm performs far better for motion $R_{\mathrm{h}}$ than for motion $R_{\mathrm{v}}$ : the depth error is 2 to 12 times higher in the latter case, and the percentage of high-confidence points is $\mathbf{2 - 3 0}$ times smaller than in the former case. This difference is mainly due to the fact that, in the extreme position, there is a larger variation of depth in the horizontal direction for motion $R_{\mathrm{h}}$. The fact that depth cannot be recovered for the normal plane and motion $R$ does not mean that this surface cannot be discriminated from a sphere, since it keeps this position only for an instant during motion $R$.

Our theoretical approach thus leads to a first prediction-namely, that motion disparity should yield more reliable depth information for motion $R_{\mathrm{h}}$ than for motion $\boldsymbol{R}_{\mathrm{v}}$.

Second, the last line of Table 1 indicates that the performance of the algorithm is quite high for motion $O$ (here the extreme position corresponds to surface tilt of $12.45^{\circ}$ in any direction), confirming the fact that this motion is optimal for the processing of structure from motion disparity (Cornilleau-Pérès \& Droulez, in press). Therefore, motion disparity should be particularly helpful as a depth cue for motion $O$.

Table 1

Results of Computer Simulations

\begin{tabular}{|c|c|c|c|c|c|c|c|}
\hline \multirow[b]{3}{*}{ Surface } & \multirow[b]{3}{*}{ Position } & \multicolumn{6}{|c|}{ Motion } \\
\hline & & \multicolumn{2}{|c|}{$\boldsymbol{R}_{\mathrm{h}}$} & \multicolumn{2}{|c|}{$R_{\mathrm{v}}$} & \multicolumn{2}{|c|}{0} \\
\hline & & \% Error & $\%$ Points & \% Error & $\%$ Points & $\%$ Error & \% Points \\
\hline Plane & median & & & & & 0.4 & 74 \\
\hline Plane & extreme & 0.6 & 59 & 7.8 & 1.7 & 0.4 & 74 \\
\hline Sphere & median & 0.6 & 58 & 1.4 & 25 & 0.5 & 74 \\
\hline Sphere & extreme & 0.7 & 58 & 5.9 & 3.2 & 0.5 & 73 \\
\hline
\end{tabular}

Note-The algorithm of stereo correspondence from optic flow has been applied on synthetic velocity fieids for different 3-D motions (see text for their definitions) and surfaces (spheres and planes) that are either tangent to a frontal plane (median position) or tilted (extreme position). \% error = average relative error performed on depth recovery. $\%$ points $=$ percentage of reliable points for which this error could be calculated. 


\section{THE QUANTITATIVE ANALYSIS OF STEREO-MOTION COOPERATION}

A quantitative analysis of stereo-motion cooperation is necessary for the interpretation of our first group of results, and for the testing of the first of our two predictions. In Experiments $1 \mathrm{~A}-1 \mathrm{C}$, we presented the surfaces in three conditions.

In Condition S ("stereo"), the surface was viewed stereoscopically and was static. The only depth cue was position disparity.

In Condition M ("motion'), the surface was moving and viewed without binocular disparity, in the sense that the images presented to the left and right eyes were both calculated as viewed from the left eye. The only depth cue was motion parallax.

In Condition MS ("motion and stereo"), the surface was viewed stereoscopically and was moving. At least two depth cues were then available: motion parallax and position disparity.

To quantify stereo-motion integration, we used two models of cue combination.

In the model of the optimal cue (or Model OC), it is assumed that the visual system can use only one depth cue in a given situation and select the optimal of the two depth cues for that situation. It predicts that the performance in Condition MS should be equal to the best performance obtained in Condition $\mathbf{M}$ or $\mathbf{S}$.

In the model of probability summation (or Model PS), if $p$ is the rate of correct responses recorded during a session, $p$ varies between 0.5 and 1 . We can therefore define a detection probability $y=2 p-1$ that ranges between 0 and 1 . If we assume that subjects give false responses at random, $y$ represents the rate of correct responses not due to chance, and it can be considered roughly as the probability of perceiving the true surface structure with the help of a given depth cue. If $y_{i}$ is the probability $y$ for a given condition $i(i=\mathrm{S}, i=\mathrm{M}$ or $i=\mathrm{MS})$, the model of probability summation predicts that $y_{M S}$ must be equal to the calculated quantity $y_{\mathrm{PS}}=y_{\mathrm{S}}+y_{\mathrm{M}}\left(1-y_{\mathrm{S}}\right)$ (see, e.g., Blake \& Fox, 1973). Model PS clearly predicts higher performance than does Model OC.

In Model PS, it is assumed that the false responses are given entirely at random by the subject. This is not true, however, and many subjects exhibit a tendency to answer "plane" more than "sphere," and vice versa. Therefore, we also considered the model of probability summation as it is proposed within signal detection theory and receiver operating characteristic analysis (Green \& Swets, 1974). After $d^{\prime}$ has been calculated for the different conditions, according to this model, $d^{\prime}{ }_{\text {MS }}$ should be equal to $d^{\prime}{ }_{\mathrm{PS}}=$ $\left(d^{\prime} \mathrm{S}+d_{M}^{\prime n}\right)^{1 / n}$, where $n=2$. Other values of $n$ correspond to different types of interactions, and the strength of motion-stereo cooperation is considered to decrease as $n$ increases (Thomas \& Olzak, 1990). This second type of model of probability summation was tested, and was found to lead to conclusions similar to those based on Model PS.

The comparison of $y_{\mathrm{MS}}$ with $y_{\mathrm{PS}}$ and $y_{\mathrm{OC}}$ yields five alternatives.
1. If $y_{\mathrm{MS}}<y_{\mathrm{OC}}$, the presence of two depth cues impairs the use of the optimal cue for the determination of 3-D structure.

2. If $y_{M S}=y_{O C}$, the visual system behaves as if it has used only one depth cue-the optimal one-in the analysis of depth.

3. If $y_{\mathrm{OC}}<y_{\mathrm{MS}}<y_{\mathrm{PS}}$, the visual system can use both cues in the analysis of a visual scene, although with poorer performance than would be predicted by Model PS.

4. If $y_{\mathbf{M S}}=y_{\mathrm{PS}}$, stereo-motion integration is described well by the model of probability summation.

5 . If $y_{M S}>y_{P S}$, there is cooperation between the two depth cues. It could be due to the presence of a third cue such as motion disparity, which is available only in Condition MS.

Note that the variable $y$ can be replaced by $p$ (rate of correct responses) in this discussion.

\section{EXPERIMENTS 1A-1C Stereo-Motion Cooperation}

\section{Method}

\section{Subjects}

The 11 subjects ( 9 women, 2 men) were 21-30 years old and had normal uncorrected vision. Ten were naive and paid volunteers, and the 11 th was one of the authors (V.C.-P.). All the subjects had a stereo acuity of better than 20 arcsec.

\section{Apparatus}

The images consisted of a set of points surrounded by a circular window that was $8^{\circ}$ of visual angle in diameter. They were displayed $72 \mathrm{~cm}$ from the subject, on a $2048 \times 2048$ vector scan display (viewing area: $29.26 \times 20.48 \mathrm{~cm}$ ), at a frequency of $15.33 \mathrm{~Hz}$. The actual resolution was $2.05^{\prime}$ of visual angle. For static stereopsis, this was improved by displaying a dot randomly in four possible positions according to its computed real coordinates; for instance, if a dot had a theoretical $x$-coordinate of 1.4 in dot-space unity, it was displayed six times in the position of $x$-coordinate 1 , and four times in the position of $x$-coordinate 2 , in random temporal order. The other technical characteristics of our experimental setup have been detailed elsewhere (Cornilleau-Pérès \& Droulez, 1989). However, instead of presenting one image, we displayed two images on the left and right of the screen, which were viewed by the left and right eyes, respectively. The centers of the circular images were located $7 \mathrm{~cm}$ on each side of the subject's sagittal axis. A black tunnel prevented lateral vision and was divided into two parts by a vertical partition located in the subject's sagittal plane. Therefore, only one image was visible to each eye. In addition, binocular fusion was helped through the use of displacing prisms. These prisms were very narrow $\left(11.5^{\circ}\right.$ base angle $)$ and did not produce any visible fringe on the stimuli.

\section{Image Computation}

Initially, for the median position on the surface, 400 dots were randomly spread over a patch larger than could be seen through the circular window, so that their density would be uniform on the display screen. Each dot position on the screen was calculated in perspective projection, as the intersection between the screen and the line joining the object dot to the observer's eye. This calculation was repeated for each position of the surface depicting its motion (46 images were devoted to an oscillation) for Conditions $M$ and MS. The sequences presented to the left and right eyes could be calculated as viewed from the actual positions of the subject's eyes (Conditions $\mathbf{S}$ and $\mathbf{M S}$ ), or as both viewed from the left eye (Condition M). 
Only image points that fell within the circular window were displayed. In the median image, the average number of dots was 205 , with a standard deviation of 10 . For the conditions involving motion, the number of dots increased from the median to the extreme image by a number that averaged less than 3 . We made sure that in all image sequences and for all types of surfaces, no contour information was available, and that our stimuli were totally deprived of any monocular depth information, except motion parallax. In Experiment 1C, we ensured, as a control, that subjects did not base their responses on static dot density

\section{Surface Motion}

In Conditions $\mathrm{M}$ and MS, the surface oscillated around a frontal axis that was tangent to the surface (motion $R$ ). The direction of this rotation axis could be vertical or horizontal (motions $R_{\mathrm{h}}$ and $R_{\mathrm{v}}$, Figure 2). Motion $R$ was composed of a rotation around the observer's eye and a frontal translation, the component of frontal translation being then in a direction orthogonal to the rotation axis. The amplitude of motion $R$ could be quantified by the total extent of the component of frontal translation $T$ expressed in unity $\Delta$ equal to the interocular distance, $6.2 \mathrm{~cm}$, or by the total extent $a$ of the rotation around the frontal axis. If $D$ is the viewing distance, $T$ and $a$ are linked by the equation $\tan (a / 2)=T /(2 D)$.

The motion amplitude was $5 \Delta$ for Subjects N.H. and F.T., and $3 \Delta$ for the 4 other subjects. After the data had been gathered from the first 2 subjects, the amplitude was diminished to obtain closer performance for Conditions $M$ and $S$. Recall that one stereoscopic image can be considered as obtained from another by a horizontal frontal translation of $1 \Delta$. The oscillation period was $3 \mathrm{sec}$, and four complete oscillations were presented on each trial.

\section{Simulated Viewing Distance}

The subject's sagittal axis and the surface intersect at point $K$ (Figure 2 ). In the median image, the surface is normal to the sagittal axis at $K$, which was stationary throughout all motions. The simulated viewing distance (between $K$ and the observer) was chosen under two constraints: (1) Stereoscopic vision is known to be highly sensitive to depth discontinuities; therefore, the simulated distance between the circular window (located $72 \mathrm{~cm}$ in front of the subject's eye) and the dots of the surface displayed in the neighborhood of this window had to remain constant in Condition S. (2) In Conditions $\mathbf{M}$ and MS, all the visible dots had to remain behind the plane of the circular window throughout the movement (to obviate perceptual conflicts).

These two constraints were satisfied by using curvature to vary the simulated viewing distance slightly. This distance ranged from 72.7 to $73.4 \mathrm{~cm}$ as curvature decreased from $3.25 \mathrm{~m}^{-1}$ to 0 . Note that because motion $R$ was a rotation of fixed amplitude in relation to an axis tangent to the surface, the position of this axis also slightly covaried with surface curvature.

The fact that responses were not biased by the disparity between outer image points and the window in Condition MS and for the extreme images, by the simulated viewing distance of the surface, and by the distance of the rotation axis in all conditions was verified in the control experiments (1B and $1 C$ ).

\section{Design and Procedure}

Each experimental session consisted of the presentation of fortyeight 12-sec image sequences. With the help of the computer keyboard, the subject indicated whether he/she had seen a planar or a spherical surface. The PCR (percentage of correct responses) was averaged over four or six sessions for the curvatures, yielding an intermediate value of that PCR. Within each session, all motion parameters were kept constant, as was the sphere curvature. Each group of sessions corresponding to a set of parameters (Conditions $S, M$, and $M S$ and two motion directions in Experiment $1 \mathrm{~A}$, three directions of the surface slant in Experiment 1B, three control conditions in Experiment 1C) was performed during the same day by each subject. The results were analyzed with Student's $t$ test. Unless otherwise specified, the level of significance was $p<.05$.

\section{Results}

\section{Experiment 1A}

\section{Stereo-Motion Cooperation}

Psychometric curves. The results obtained for Conditions $S, M$, and MS are shown in Figures 3 and 4, for motions $R_{\mathrm{h}}$ and $R_{\mathrm{v}}$, respectively. Where the standard deviation is indicated in brackets, the PCR has been averaged over four to six sessions (as mentioned above, this was done only for curvatures yielding intermediate values of the PCR). In order to restrain the discussion of the results to close-to-threshold levels, we shall consider only the two lower curvatures tested for each subject (these

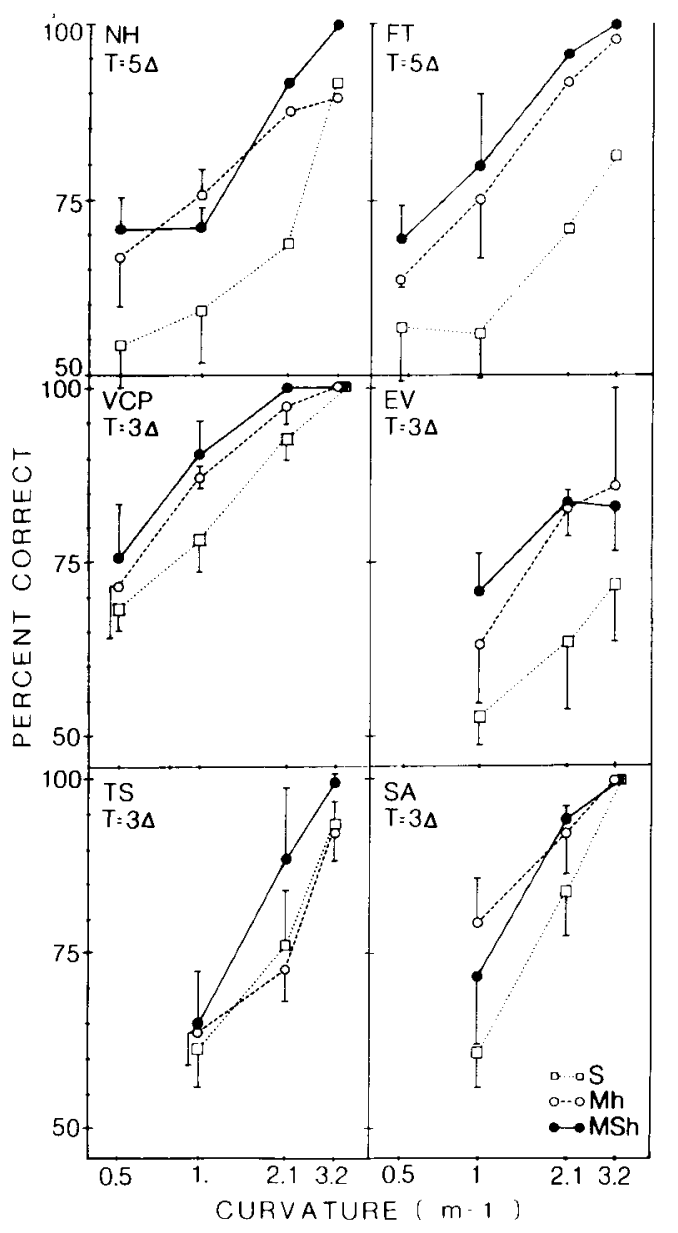

Figure 3. Stereo-motion cooperation, psychophysical curves. Abscissae: sphere curvature (inverse of sphere radius). Ordinates: percentages of correct responses in a task of discrimination between planar and spherical patches. When the data point is the average of several sessions (between four and six), the standard deviation is indicated with a bracket. These results have been obtained for motion $\boldsymbol{R}_{\mathrm{h}}$, for three conditions: $\mathrm{S}$ (squares), $\mathrm{M}$ (empty circles), and MS (filled circles). The initials of the subject as well as the motion amplitude (see text) are indicated on each panel. 


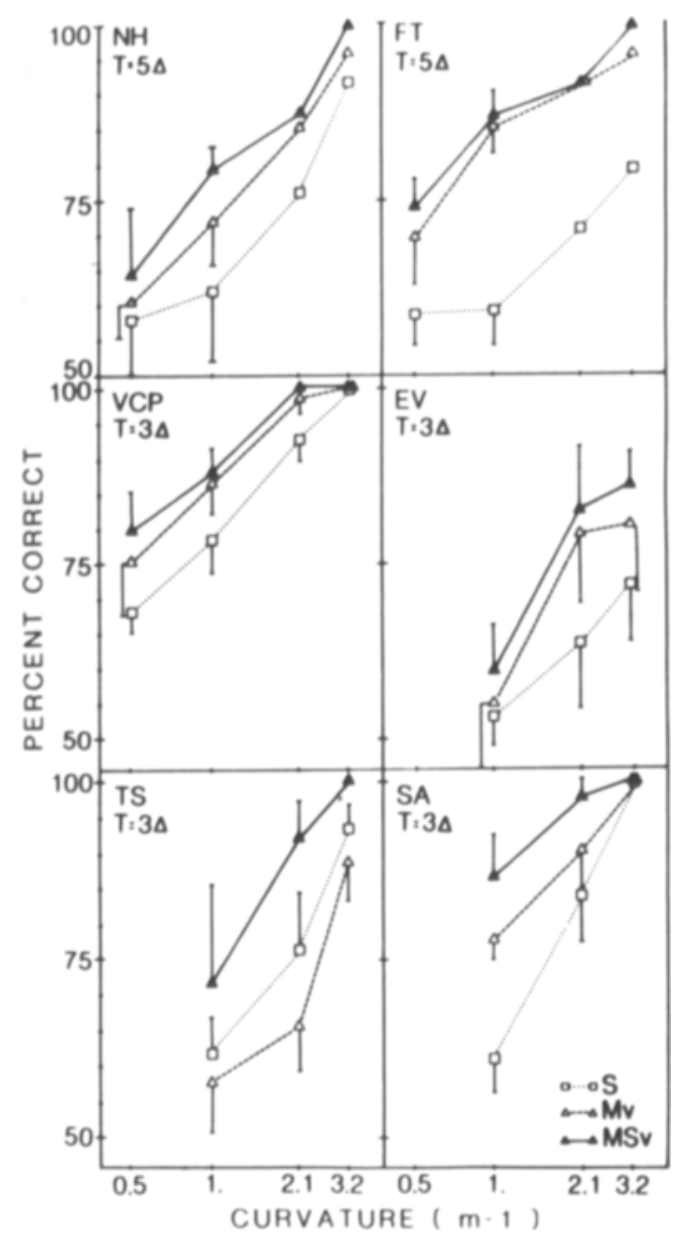

Figure 4. Same as Figure 5, except that the results were obtained for motion $\boldsymbol{R}_{\mathrm{y}}$ and the circles have been replaced by triangles.

curvatures yielded PCRs that were intermediate between $50 \%$ and $100 \%$ in all conditions).

The discrimination task was generally easier to perform from motion parallax than from stereopsis. All the subjects except T.S. performed better in Condition $M$ than in Condition $\mathbf{S}$, and this was significant for 9 of the 20 points ( 5 subjects, 2 curvatures, 2 motion directions) considered. For Subject T.S., Condition $S$ yielded a better performance than did Condition $M$ in 3 points out of 4 .

The subjects' performance was generally optimal when the two depth cues were present. This was true for all subjects, curvatures, and motion directions, except for 2 of 24 points (Motion $R_{\mathrm{h}}$, Subjects S.A. and N.H., $c=$ $1 \mathrm{~m}^{-1}$ ). However, among the 22 other points, the increase of performance in Condition MS, yielding the highest PCR in comparison with Condition $M$ or $S$, was found to be significant only for 2 points (motion $R_{v}, c=$ $2.125 \mathrm{~m}^{-1}$; Subject S.A., $N=6, t=3.84, p<.02$; Subject T.S., $N=6, t=7.59, p<.01$ ).

These results indicate globally that the model of the optimal cue underestimates the stereo-motion cooperation and therefore our results for motion $R_{\mathrm{h}}$ and motion $R_{\mathrm{v}}$ do not seem to fall within Alternatives 1 and 2 listed above.
Influence of the direction of motion. The percentage of correct responses of the 6 subjects in Conditions $M$ and MS is shown for motions $R_{\mathrm{h}}$ and $\boldsymbol{R}_{\mathrm{v}}$ in Figure 5, for the two curvatures yielding intermediate performance. No systematic anisotropy can be observed for Condition $M$ or MS. However, there is a general tendency to prefer motion $R_{\mathrm{h}}$ in Condition $\mathrm{M}$ (for 9 of the 12 experimental points corresponding to each panel of Figure 5 , this being significant for 1 point; Subject E.V., $c=1 \mathrm{~m}^{-1}, N=$ $5, t=2.9, p<.05$ ), whereas this tendency is reversed in Condition MS (for 8 of 12 points, the task is easier for motion $R_{\mathrm{v}}$, this being significant for 3 points; Subject S.A., $c=2.125 \mathrm{~m}^{-1}, N=6, t=4.983, p<.01$, and $c=1 \mathrm{~m}^{-1}, N=5, t=7.38, p<.01$; Subject N.H., $c=1 \mathrm{~m}^{-1}, N=4, t=4.38, p<.05$ ). This reversal is mainly due to Subjects T.S. and S.A.; when stereopsis is added to motion parallax, these 2 subjects clearly exhibit a change in the motion direction of maximal performance, in the sense that motion $R_{\mathrm{v}}$ yields optimal stereo-motion cooperation. On the other hand, no such systematic effect can be seen for the 4 other subjects.

Quantitative analysis of stereo-motion cooperation. In Figure 6, we plotted the PCR obtained in Condition MS as a function of the PCR predicted by the models of op-

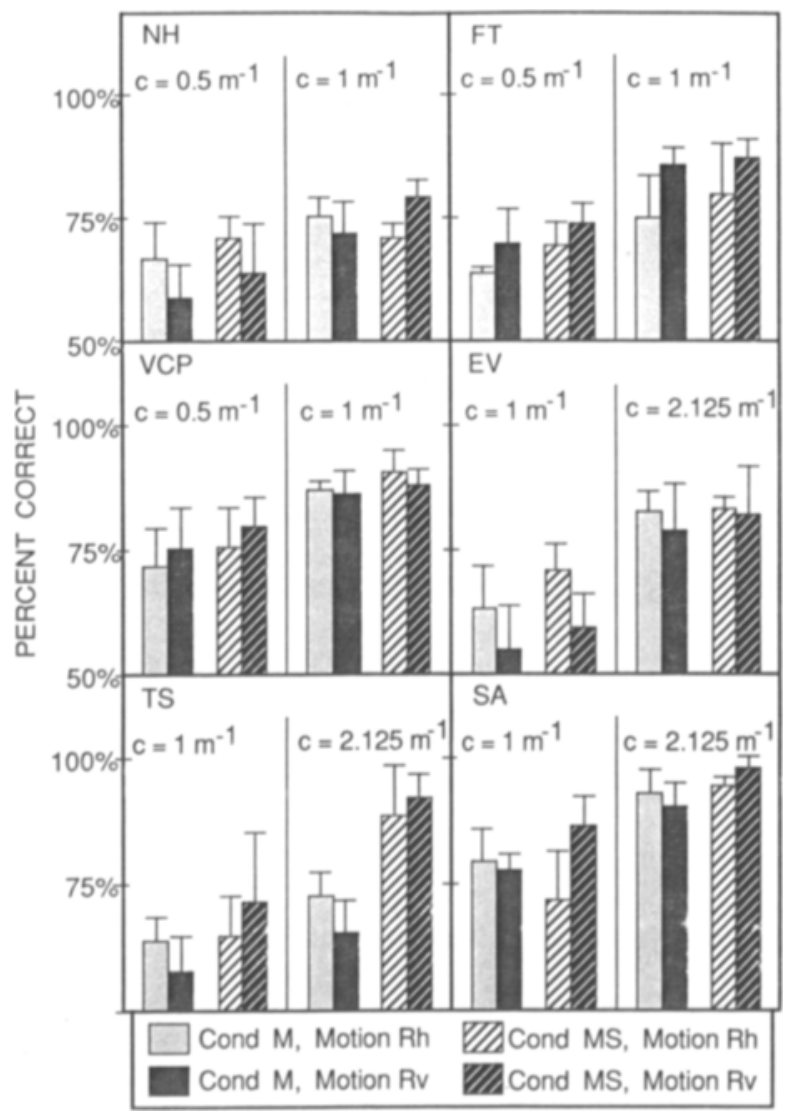

Figure 5. Comparison between motion $\boldsymbol{R}_{\mathrm{h}}$ and motion $\boldsymbol{R}_{\mathrm{v}}$. For each subject and the two curvatures yielding intermediate performance in all conditions, the percentage of correct responses is compared for motions $\boldsymbol{R}_{\mathrm{h}}$ and $\boldsymbol{R}_{\mathrm{v}}$, in Conditions $M$ and MS. 
A. MODEL OC

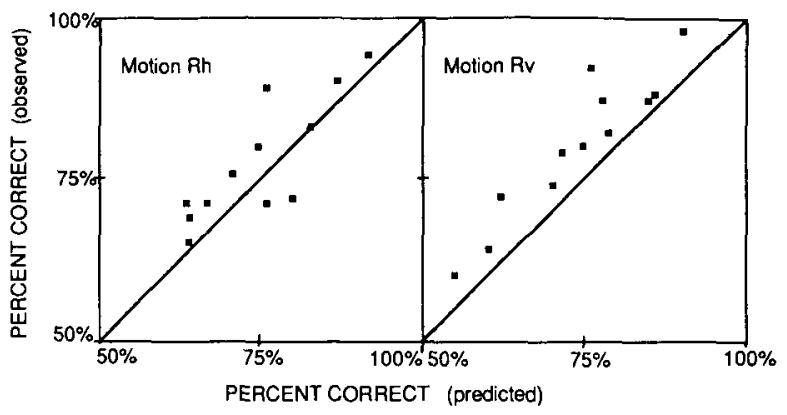

B. MODEL PS

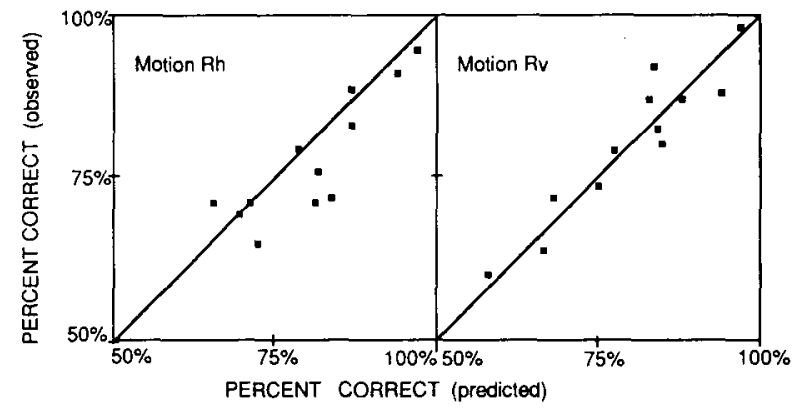

Figure 6. Comparison of the observed stereo-motion cooperation relative to Models $\mathrm{OC}$ and PS. In each panel, the percentage of correct responses (PCR) observed for Condition MS is plotted as a function of the PCR predicted by a model from the responses observed in Conditions $M$ and $S$. The type of model and the motion $\left(R_{h}\right.$ or $\boldsymbol{R}_{\mathrm{v}}$ ) involved are indicated within each panel. Each point is the result for 1 subject and one curvature. (A) Comparison for Model OC. (B) Comparison for Model PS.

timal cue (OC) and probability summation (PS). Figures 6A and 6B correspond to Models OC and PS, respectively. If the models fitted the data exactly, the points would be located on the diagonal lines of the panels. Should they overestimate stereo-motion cooperation, this line would be located above the points.

Since most of the points are located above the diagonal line in Figure 6A, Model OC tends to underestimate the actual performance. Alternatively, Figure 6B indicates that Model PS does not systematically under- or overestimate the curvature sensitivity.

To compare stereo-motion cooperation for motions $R_{\mathrm{h}}$ and $R_{\mathrm{v}}$, we quantified the difference between the observed and predicted performance. We calculated the difference between observed and predicted PCRs for 12 values for each motion ( 6 subjects, 2 curvatures); we call this distribution the remainders. If the mean of the remainders is positive, the performance is better than that predicted by the model. From the remainder distribution, one can locate the observed performance relative to Models OC and PS. We found that for motion $R_{\mathrm{h}}$, the level of stereomotion cooperation is located between Models OC and PS (significantly lower than predicted by Model PS, $N=$ $12, t=2.33, p<.05$ ), and that for motion $R_{\mathrm{v}}$, stereomotion integration was underestimated by the two models (this is significant for Model OC, $N=12, t=5.23, p<$ $.01)$. Overall, this suggests that stereo-motion cooperation is more efficient for motion $R_{\mathrm{v}}$ than for motion $R_{\mathrm{h}}$. However, if we take any of the two models as a reference, the difference between the two movements is not significant $(N=12$; Model OC, $t=1.83$; Model PS, $t=1.95)$.

As mentioned above, we also applied a receiveroperating characteristic analysis to our results. A prerequisite for this is that the PCR obtained for the sphere should increase as the percentage of false alarms increases (when the subject answers "sphere" after viewing a plane). We could not find a systematic positive correlation between these percentages when they were measured from different trials in similar conditions. It was therefore impossible to determine which type of distribution could be used for this analysis. Nevertheless, the use of Gaussian distributions of equal variance led us to search for the value of $n$ that would minimize the quadratic difference between the distribution of the observed $d^{\prime}$ for Condition MS, and the distribution $d_{n}^{\prime}=\left(d_{M}^{\prime \prime}+d^{\prime \prime} \mathrm{S}\right)^{1 / n}$. Following Thomas and Olzak (1990), the limits $n_{1}$ and $n_{2}$ of the $95 \%$ confidence interval of $n$ are found by testing the difference between the distributions $d_{n}^{\prime}$ and $d^{\prime}$ for different values of $n$, against a value of zero, using Student's $t$ test with $\alpha=$ .05 . We found that $n=2.20$ for motion $R_{\mathrm{h}}$, with a $95 \%$ confidence interval $(1.335,5.025)$, and 1.41 for motion $R_{\mathrm{v}}$, with a $95 \%$ confidence interval $(0.945,1.88)$. This agrees with the previous conclusion, in the sense that stereo-motion integration tends to be better for motion $\boldsymbol{R}_{\mathrm{v}}$ than for motion $R_{\mathrm{h}}$ (the value of $n$ found for $R_{\mathrm{v}}$ is smaller than the value of $n$ found for $R_{\mathrm{h}}$ ). This tendency is not significant, since the value of $n$ found for motion $R_{\mathrm{v}}$ belongs to the $95 \%$ confidence interval of motion $R_{\mathrm{h}}$. In addition, a model of probability summation corresponding to $n=2$ tends to overestimate stereo-motion integration for motion $\boldsymbol{R}_{\mathrm{h}}$ but not for motion $\boldsymbol{R}_{\mathrm{v}}$.

The individual data concerning this anisotropy is plotted in Figure 7. For each subject and each curvature, the difference between performance in Condition MS and predicted performance from Model PS is compared for motions $R_{\mathrm{h}}$ and $\boldsymbol{R}_{\mathrm{v}}$. Similarly to Figure 5 , Figure 7 confirms that the failure for assessing a significant value to this anisotropy is due to the interindividual variations. It is observed clearly for Subjects S.A. and T.S., whereas other subjects exhibit anisotropies that are either small or vary in direction.

In conclusion, the results for motion $R_{\mathrm{h}}$ correspond to Alternative 3, whereas the results for motion $R_{\mathrm{v}}$ are described better by Alternative 4 or 5 . However, because of a high interindividual variability, this anisotropy is not significant.

\section{Experiment 1B: The Influence of Surface Slant on the Detection of Curvature in Condition $S$}

During motion $R$, the slant of the surfaces, measured in relation to a frontal plane, varies in the horizontal direction for motion $R_{\mathrm{h}}$, and in the vertical direction for motion $R_{\mathrm{v}}$. If the detection of surface curvature from position 


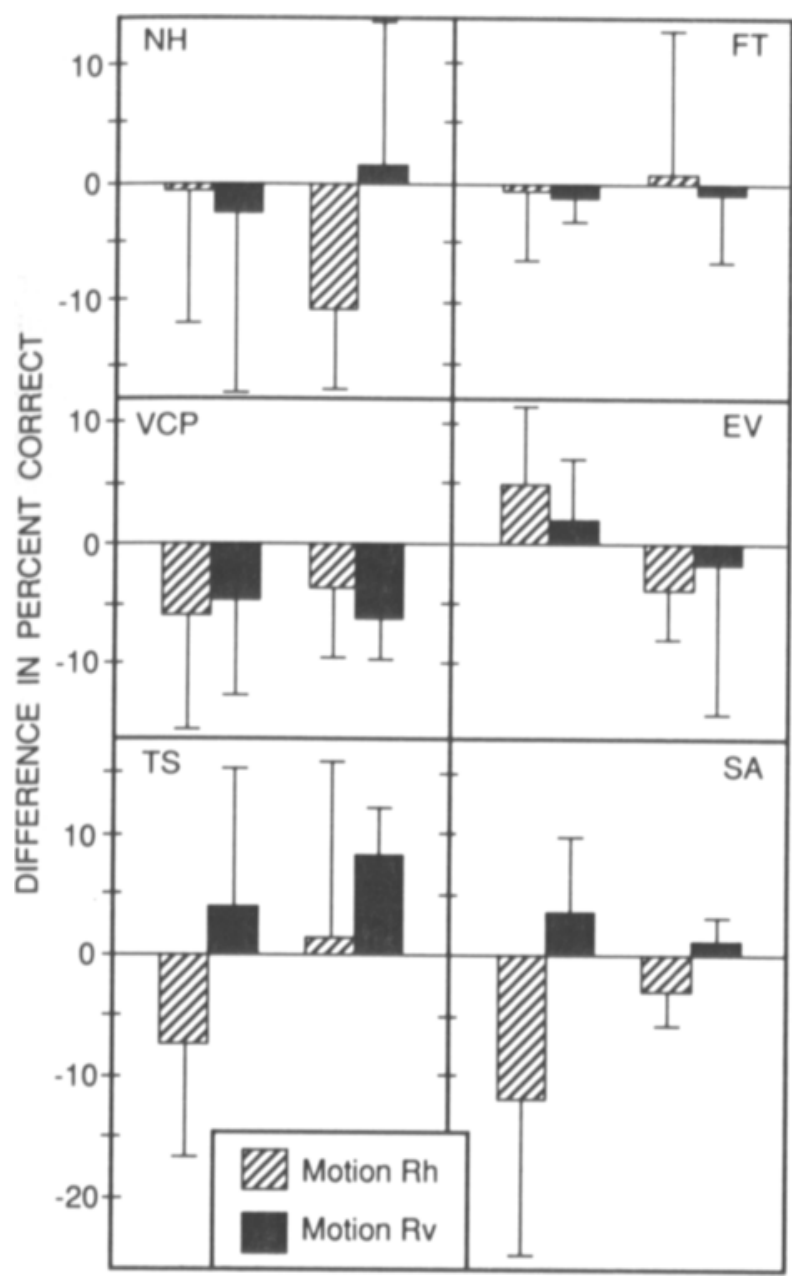

Figure 7. Difference between performance observed in Condition MS and predicted by Model PS. For each subject, the difference of PCR has been indicated for the two lower curvatures tested and the two directions of motion. A negative value means that the performance is lower than that predicted by Model PS.

disparity depended on the direction of slant of the surface, it could induce an anisotropy between the two directions of motion $R$ in Condition MS. Since the extreme values of slant are $-12.45^{\circ}$ and $12.45^{\circ}$ for motion amplitude $5 \Delta\left(0^{\circ}\right.$ correspond to the normal plane), we compared the capacity to detect curvature in Condition $S$ for three orientations of the surfaces: (1) the surface normal to the sagittal axis (Condition S); (2) the surface tilted by $12.45^{\circ}$ in the vertical direction (Condition Sv); and (3) the surface tilted by $12.45^{\circ}$ in the horizontal direction (Condition Sh).

Figure 8 shows the PCRs obtained for 4 subjects and $c=2.125 \mathrm{~m}^{-1}$ or $1 \mathrm{~m}^{-1}$. Each point is the mean of five sessions. No significant difference could be found between any two of the three conditions for each subject $(t<2.07$, $N=5$ ), and no systematic tendency to prefer one or the other direction of slant appears in Figure 8. Therefore, the difference in slant direction cannot account for any anisotropy in relation to the role of motion direction in stereo-motion cooperation.

\section{Experiment 1C: The Influence of}

2-D Velocity, Acceleration Magnitudes, and Static Dot Distribution

Through computer calculation, we found that the amplitude of the velocity and acceleration of the dots covaried with curvature. For the plane and the sphere of curvature $1 \mathrm{~m}^{-1}$ (motion $3 \Delta$ and Condition $\mathrm{M}$ ), we averaged, over 100 image sequences, the mean velocity magnitude over all dots and all images, the maximum velocity magnitude in each trajectory of each dot, the mean acceleration magnitude, and the maximum acceleration magnitude, and we found that these four variables were statistically different for the plane and the sphere.

In addition, the images of the sequences present spatial variations of dot density that are different for the plane and the sphere. This difference is null for the median images and maximum for the extreme images (displaying the slanted surfaces).

We therefore performed a control experiment that involved three conditions: Condition $\mathbf{M h}$ (Condition $\mathbf{M}$, Motion $\boldsymbol{R}_{\mathrm{h}}$, with fixed simulated viewing distance for the sphere and the plane), identical to the paradigm in Experiment 1A; Condition Dist, which was similar to Condition Mh, except that the viewing distance was randomly chosen between 63.3 and $83.3 \mathrm{~cm}$; and Condition Stat, which was similar to Condition Mh, except that the median image, a black image, and the extreme image were displayed successively for 3,2 , and $3 \mathrm{sec}$.

For Condition Dist, the interval $(63.3,83.3 \mathrm{~cm})$ was chosen as being centered on the mean viewing distance of the 1 -m-radius sphere $(73.266 \mathrm{~cm})$ and the plane $(73.396 \mathrm{~cm})$ from Experiment 1A. A computer calculation showed that it contained a subinterval for which each

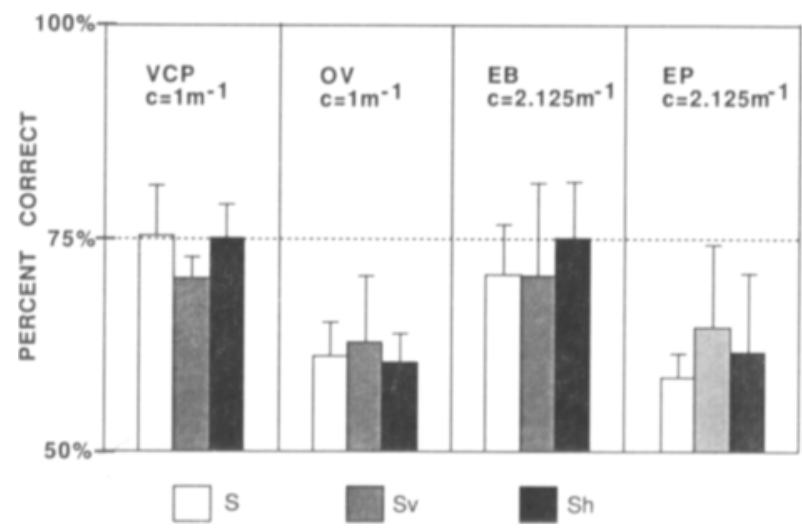

Figure 8. Control experiment: influence of the surface slant on the curvature detection in stereo vision. The surface is stationary. Ordinate: percentage of correct responses. The initials of the subject and the value of the curvature are indicated on each panel. Each bar is the mean of five sessions. White: Condition S; the surfaces are normal to the sagittal axis. Gray: Condition Sv; the surfaces are tilted by $12.45^{\circ}$ in the vertical direction. Dark: Condition Sh, the surfaces are tilted by $12.45^{\circ}$ in the horizontal direction. 


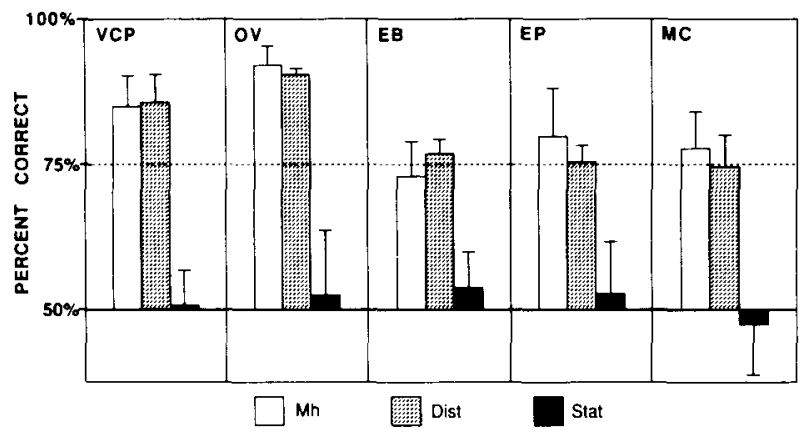

Figure 9. Control experiment: influence of image velocity and acceleration on the detection of curvature. Percentages of correct responses obtained in three conditions: Mh (motion parallax, from motion $R_{h}$ ), Dist (the same as Mh, except that the simulated viewing distance varies), and Stat (the same as $M h$, except that only the median and extreme images are displayed). The subject's initials are indicated on each panel. Each bar is the mean of six sessions. Curvature $=1 \mathrm{~m}^{-1}$.

of the four variables above shared a common range of values for the plane and the sphere. In addition, for more than $55 \%$ of the stimulus surfaces, these four variables took their values within this subinterval. Therefore, by randomly varying the viewing distance, we ensured that the four variables were uncorrelated with surface curvature for more than $55 \%$ of the stimuli, and we weakened their potential use for curvature detection considerably.

For Condition Stat, the interstimulus interval of $2 \mathrm{sec}$ was chosen so that no movement was perceived between the two images (this is well outside the domain of the short-range process). In addition, we carefully explained to the naive subjects what the two images were meant to represent.

As indicated in Figure 9, the 5 subjects tested in this experiment performed similarly in Conditions $\mathrm{Mh}$ and Dist. The difference in PCR between Conditions Mh and Dist was never significant $(t<1.58, N=6)$. This difference remained similar for each subject when only the responses obtained within the subinterval of the common range of the variables were taken into account. The maximum decrease of PCR from Condition Mh to Condition Dist was then $2.9 \%$, whereas it should have reached about $25 \%$ if a subject had based his/her responses on any of the variables listed above. The first conclusion of this control experiment was therefore that the four variables played a secondary role in the discrimination task. In particular, they could not be considered as possible artifacts for explaining the better performance in Condition $M$ than in Condition $S$ in Experiment $1 \mathrm{~A}$.

A second conclusion was that we could also discard the simulated distance between the viewer and the surface, or between the viewer and the rotation axis, as a possible artifact, since this variable was not correlated to surface curvature in this experiment.

Figure 9 also shows that Condition Stat yielded performance that was not statistically different from chance level $(t<1.53, N=6)$. This was true even when the responses of the 5 subjects were pooled together $(t=1.06$, $N=30$ ). The spatial variations of the dot density in each separate image of the sequences were therefore not sufficient for subjects to perform the task of curvature detection.

\section{EXPERIMENT 2 Is Motion Disparity a Depth Cue?}

The methods for the second set of experiments were similar to those in the first set, except that the left and right dot distributions were decorrelated. The stereoscopic image pairs were calculated as in Experiments 1A-1C, but only half the dots (randomly chosen) remained on the left image, while the other half remained on the right image. We therefore obtained two image sequences, viewed from the left and right eyes, of the same moving surface, which was defined by different sets of dots according to the destination of the sequence (left or right eye).

The stimuli thus obtained involved both motion parallax and motion disparity as depth cues, and they will be referred to here as $M P D$ stimuli (for "motion parallax and disparity"). To obtain a control stimulus that presented the same amount of motion parallax, but no motion disparity (MP stimulus), we designed the same kind of stereo sequences, except that the images presented to both eyes were calculated as if seen from the left eye.

Binocular fusion was ensured by the presence of the circular window and the point $K$ (the intersection of the surface and sagittal axis) as a fixation mark, which was more luminous than the other dots. The absence of saccadic eye movements was checked by the experimenter, who watched EOG signals on an oscilloscope screen and canceled the trial when large saccades occurred.

First, it should be mentioned that the decorrelation between the left and right images did not bother the subjects at all. Actually, all the observers, including several researchers in visual sciences, who saw the stimuli did not notice it. No systematic statistics were performed, but a pilot experiment indicated that the performance obtained for stereo-decorrelated images was not modified by the decorrelation, for motion $R$ and several curvatures ranging between 1 and $10 \mathrm{~m}^{-1}$.

The surfaces were rotated sinusoidally around the subject's sagittal axis (motion $O$ ), with a total amplitude of $51^{\circ}$ and a period of $3 \mathrm{sec}$, for $12 \mathrm{sec}$. The curvature of the sphere was $10 \mathrm{~m}^{-1}$, and the distance in depth between the nearest and the farthest points of the sphere was $1.4 \mathrm{~cm}$, which corresponded to a binocular disparity of about $0.1^{\circ}$. In spite of this large disparity, the PCRs of 3 subjects were very close to chance level in Conditions MP and MPD. In particular, we could not find any significant difference of performance between Conditions MP and MPD (Subject V.C.-P., $N=10, t=0.96$; Subject T.S., $N=6, t=0.43$; Subject S.A., $N=6, t=$ 0.21 ). Thus, we could not demonstrate the use of motion disparity as a cue for the perception of 3-D structure. 


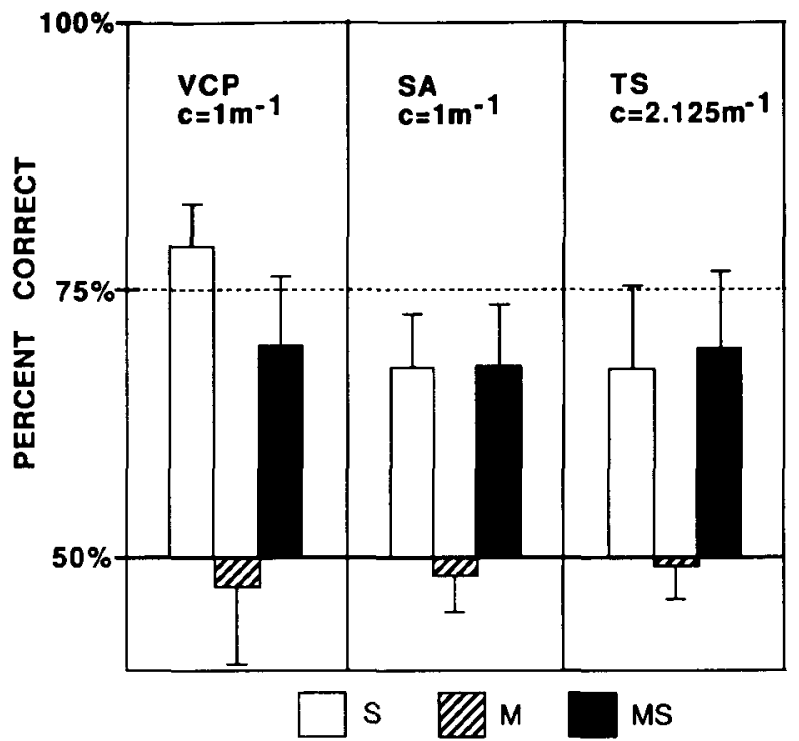

Figure 10. Stereo-motion cooperation for motion $O$. Ordinates: percentages of correct responses. Each bar is the mean of six sessions performed in Conditions S, M, and MS. On each panel are indicated the subject's initials and the sphere curvature.

A first explanation for this result is that the visual system might be unable to process structure from motion disparity in the absence of position disparity. In our experiment, it was implicitly assumed that the visual system could interpolate the function $f$ (roughly equal to the vertical component of image velocity) in order to establish correspondence between left and right image points. That the visual system is able to make an interpolation of the velocity field has been suggested by many experiments on the perception of 3-D structure from motion (Husain, Trewe, \& Andersen, 1989). However, it could be that this interpolation is not precise enough to process motion disparity as proposed in our theoretical scheme. Another explanation is that the determination of the epipolar lines is a prerequisite for the motion disparity to be used in stereo correspondence. It has been suggested that cyclovergence eye movements are involved in the binocular fusion process (Rogers \& Howard, 1991). Because of their property of symmetry, the window and the central point of the image could not drive this cyclovergence properly, and this could impair the use of motion disparity.

In a last trial to demonstrate the use of motion disparity as a depth cue, we replicated Experiment $1 \mathrm{~A}$ with Motion $O$ in Conditions $\mathrm{S}, \mathrm{M}$, and MS. Because motion $O$ provides very little motion parallax to each eye (it is a rotation around an axis passing through the middle of the interocular segment; i.e., it is very close to a pure rotation around each eye), a possible improvement of performance from Condition $S$ to Condition MS could be interpreted as due to the use of motion disparity. We used sphere curvatures yielding a PCR near threshold for Condition S. These curvatures are indicated on each panel of Figure 10, along with the subject's initials. Each point is the mean of six sessions.

Figure 10 confirms that the motion parallax elicited by motion $O$ was not sufficient to allow the detection of curvature (Condition $M$ ), since performance is near chance level. The performance measured for Condition $S$ was near threshold, and it remained unchanged in Condition MS for Subjects T.S. and S.A. On the other hand, for Subject V.C.-P., the PCR decreased significantly $(p<.05)$ from Condition $S$ to Condition MS $(N=6, t=2.73)$.

Finally, this experiment also failed to demonstrate the use of motion disparity as a depth cue. However, it provided additional information with respect to Experiment $1 \mathrm{~A}$-namely, that when motion parallax is very weak, stereo-motion integration is described by Alternative 1 or 2 , corresponding to a null or even negative interaction between the two depth cues.

\section{DISCUSSION}

\section{Summary of the Results}

Our results lead to the following conclusions:

1. In our experimental conditions, the motion parallax due to motion $R$ (a rotation around a frontal axis tangent to the surface) yielded a sensitivity to surface curvature that was comparable, although generally superior (for 5 of 6 subjects) to the sensitivity measured when position disparity is the only depth cue.

2. The cooperation between motion parallax and position disparity in the perception of 3-D structure depends on the type of 3-D motion of the object under analysis. For motion $R$, which involved a large component of frontal translation and allowed the perception of 3-D structure from motion parallax, the two depth cues had a positive interaction, in the sense that curvature sensitivity was higher in the presence of them both, than when only one of them was available. For motion $O$, which elicited litthe motion parallax, the presence of motion tended to leave unchanged or to impair the detection of surface curvature from position disparity.

3. Stereo-motion integration depends on the direction of the motion, with respect to the interocular direction. For motion $R$, it tended to be more effective when the frontal translation was vertical (and the rotation horizontal), rather than horizontal. In particular, the model of probability summation was close to the results in the former case, whereas it overestimated stereo-motion integration in the latter. However, owing to interindividual differences, this anisotropy was not found to be significant, and it will have to be confirmed in further experiments.

4. We could not find evidence of a use of motion disparity in the stereo-motion integration, at least in the way described by the theoretical scheme. First, the anisotropy relative to the direction of motion $R$ tended to be inverse to that predicted by the scheme. Second, we failed to find any proof of the use of motion disparity as a depth cue for motion $O$. 
The Visual Sensitivity to Depth From

Position Disparity or Motion Parallax

Rogers and Graham $(1982,1985)$ measured the sensitivity to depth for static corrugated surfaces observed in stereoscopic vision by a stationary observer, or in monocular vision during head translations. Their results indicate a sensitivity to depth that was higher in the former case (stereopsis) than in the latter (motion parallax). This contrasts with 5 of our 6 subjects' being more sensitive to depth from motion than to depth from stereopsis. Before concluding that this represents a discrepancy, we must compare separately the sensitivity to depth from stereo obtained by the two groups of authors, and then the sensitivity to depth from motion. The surfaces used by Rogers and Graham had sinusoidal depth corrugations (1982) or difference of Gaussian profiles (1985). Since our surfaces had a nonperiodic depth profile, we will restrain the comparison to Kogers and Graham's (1985) study. For reasons given in the Appendix, it makes sense to take into account the depth thresholds obtained by Rogers and Graham for a width of the difference of Gaussians (DOG; distance between two troughs) of $8^{\circ}$ or $16^{\circ}$. Because the motion used by these authors was horizontal, with an amplitude of $13 \mathrm{~cm}$, we will consider only the results that we obtained for a motion amplitude $3 \Delta$ (4 subjects) in Condition $\mathrm{M}$, motion $R_{\mathrm{h}}$. Finally, the frequency of the motion was similar in the two studies (about $0.5 \mathrm{~Hz}$ for Rogers \& Graham, $0.33 \mathrm{~Hz}$ for us).

In Figure 11 are plotted curvature thresholds interpolated from Figures 3 and 4 and converted into equivalent disparity (see the Appendix for details, along with the thresholds found by Rogers and Graham (1985). For depth from motion, these thresholds are very similar in the two studies, whereas stereopsis yielded a sensitivity to depth that was clearly better in Rogers and Graham's experiment, in comparison with our results. As discussed in the Appendix, the difference observed in stereo thresholds is most probably due to the difference in the dot distributions, which were dense in Rogers and Graham's studies but very sparse in ours. Indeed, the interactions between points of different binocular disparities can be of various types (attraction or repulsion in depth), depending on the viewing angle between them (Westheimer \& Levi, 1987). Interestingly, thresholds are very similar in the motion condition, although Rogers and Graham used self-motion rather than object motion as a source of parallax. Note that the weak influence of dot density on the perception of depth from motion has been reported in many studies (see Petersik, 1980) and was confirmed in one of our pilot experiments.

Finally, the main difference between the results obtained by Rogers and Graham (1985) and those in our Experiment 1 A concerns stereo thresholds. The comparison of the two studies does not underline any discrepancy; rather, it raises questions concerning the comparative role played by spatial parameters (such as dot density) in the perception of structure from motion or from binocular disparity.

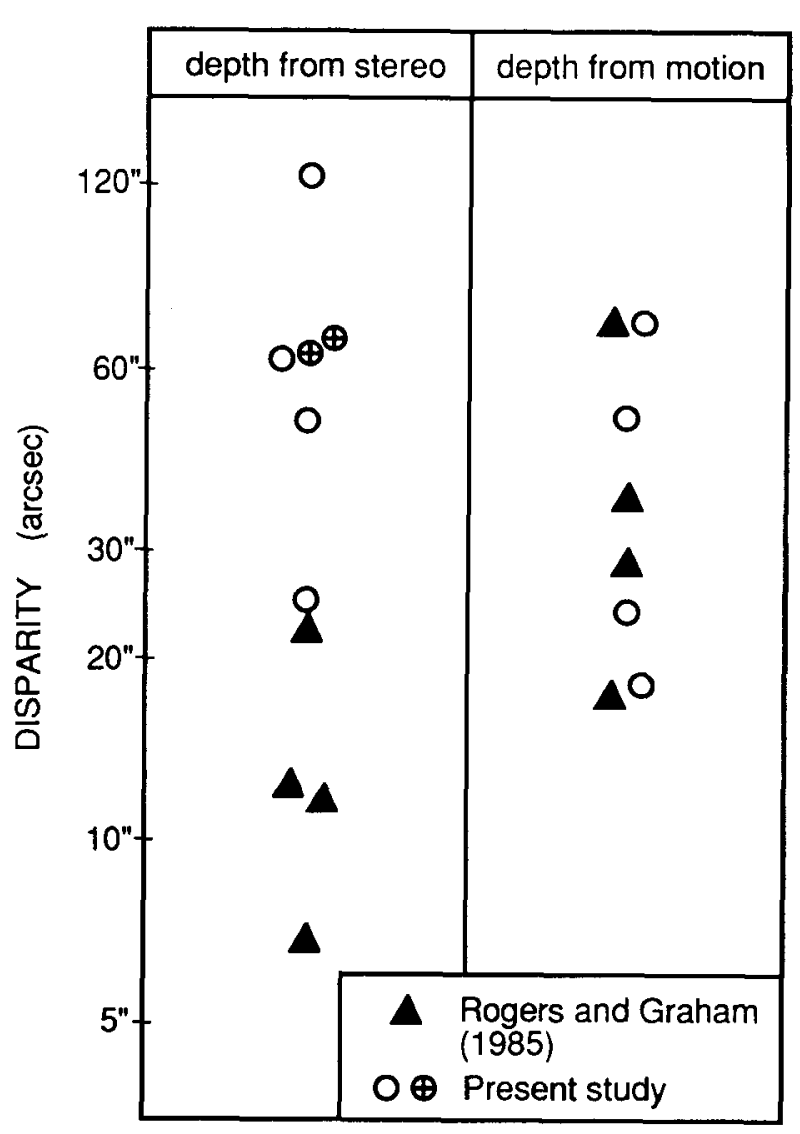

Figure 11. Comparison of thresholds for depth perception from stereopsis (left) or motion parallax (right). Triangles: results from Rogers and Graham (1985), 2 subjects, width of the difference of Gaussians: $8^{\circ}$ and $16^{\circ}$. Circles: results from the present study. Empty circles correspond to thresholds obtained for the 4 subjects who were tested for motion amplitude $3 \Delta$. Circles with a cross indicate stereo thresholds for the 2 other subjects. Ordinates: thresholds in equivalent binocular disparity.

\section{Stereo-Motion Cooperation}

Our results clearly show that the two depth cues yield better performance than one, when the motion of the surface involves motion parallax (motion $R$ ). However, when this motion yields no motion parallax but only a global movement of the image (motion $O$ ), motion does not improve, and it can even perturb, depth perception in binocular viewing. Indeed, a complete analysis of stereo-motion cooperation must take into account the impairment of the detection of position disparity in the presence of retinal image motion. This effect has been described by Westheimer and McKee (1975) as taking place above $1 \% / \mathrm{sec}$ for oblique motion, and $2.5 \% \mathrm{sec}$ for horizontal or vertical motion. In Experiment 2 (motion $O$ ), the mean dot velocity increased from zero in the image center to $2.37^{\circ} / \mathrm{sec}$ in the outer border, and the resulting retinal image motion could thus explain the impairment of the performance in Condition MS (motion and stereopsis) for 
Subject V.C.-P. On the other hand, stereo matching should not be affected by image motion for motion $R$, where all dot velocities were lower than $0.5 \% \mathrm{sec}$. Therefore, the effect described by Westheimer and McKee is not likely to mask stereo-motion cooperation for motion $R$, and it cannot explain our failure to demonstrate the role of motion disparity for this motion.

The theoretical approach (Droulez \& Cornilleau-Pérès, 1990) demonstrates that the key parameter for the perception of structure from motion is the frontal translation involved in the 3-D motion (this does not mean that a pure frontal translation is the best 3-D movement for depth perception, but simply that a movement devoid of frontal translation does not yield the perception of structure from motion). Similarly, the perception of depth from position disparity is due to the frontal translation that transforms the left view into the right view. Therefore, it would not be surprising that stereo-motion cooperation depends on the direction of the frontal translation involved in the object motion. No systematic anisotropy of such type could be exhibited from our results, and the mean tendency to perform better with a vertical, rather than horizontal, direction of frontal translation was not confirmed for all subjects. Nevertheless, this tendency is opposite to the predictions of our theoretical scheme, which argues against its physiological validity.

A type of complex interaction between motion parallax and stereopsis has been documented by Nawrot and Blake (1991). As a transparent dotted sphere seen in orthogonal projection is rotating around its vertical axis, the perceived direction of rotation undertakes reversals in monocular viewing. The stereoscopic presentation of the same stimulus eliminates the ambiguity that is relative to motion direction. After prolonged viewing of such a stimulus, the rotation direction in monocular vision is not ambiguous anymore, but opposite to the direction of rotation of the adaptation sphere. After testing many control conditions, the authors concluded that this effect was local, in the sense that the adaptation and test stimulus have to be presented on the same part of the visual field, but global, in the sense that it cannot be due to the local adaptation of neurons selective to velocity and binocular disparity.

Rogers and Collett (1989) also described interactions between binocular disparity and motion parallax by presenting discrepant motion parallax and position disparity signals. In particular, when the corrugations of a surface are specified by a position disparity that is not coherent with motion parallax, the perceived 3-D motion of the surface is modified. This result suggests that the depth information provided by position disparity may deeply influence the processing of 3-D motion. It is also supported by Gogel's demonstration (1980) that the slant of a bar, when specified by binocular disparity, influences the perceived 3-D motion of this bar, concomitant with head movements of the observer.

Rogers and Collett (1989) found that, in the absence of binocular disparity between the left and right images of the translating surface, the amount of perceived depth in binocular viewing was about half the amount of perceived depth in monocular viewing. It is as if a positiondisparity signal of zero depth was averaged with the signal of depth elicited by motion parallax. Indeed we verified that the impression of depth was more vivid in monocular than in binocular viewing in our experimental setup when motion parallax was the only depth cue. However, our experiments (Cornilleau-Pérès \& Droulez, 1989, Experiment 5) showed that the discrimination between cylindrical and planar surfaces was not affected by monocular, rather than binocular, viewing when motion parallax was the only depth cue. This apparent contradiction might merely reflect the fact that Rogers and Collett studied supraliminar levels (the subject had to judge the amount of depth), whereas we measured thresholds for depth detection. It might therefore be that the presence of null position disparities influences the sensitivity to depth and the magnitude of apparent depth differently.

\section{Motion Disparity and the Perception of 3-D Structure}

From our results, the use of motion disparity as a depth cue remains unclear, and the physiological validity of our theoretical scheme for the processing of motion disparity is seriously questioned. Establishing analogies with the visual processing of position disparities, we propose several reasons to account for this conclusion.

First, the function $f$ on which the correspondence process in our scheme is based varies smoothly over the image in our stimuli. From the experiments of Bülthoff and Mallot (1987), we know that steep variations of the luminous intensity $I$ are essential for the stereo correspondence process. Moreover, the studies of Marr and Poggio (1979) and Mayhew and Frisby (1981) even suggest that the distribution of particular intensity variations over the image serves as the basic input to stereo correspondence. Steep variations of the function $f$ might also be optimal for the perception of 3-D structure from motion disparity. To answer this question, future experiments will have to involve high contrasts in vertical velocity. Second, to a good approximation, eye movements do not modify the coding of the input to stereopsis-that is, the luminous intensity 1 . On the contrary, at a given retinal point, the function $f$ depends on the image velocity, the retinal eccentricity, and also the direction of gaze relative to the sagittal axis (the angle $\theta$ in Equation 2 depends on the latter two parameters). This might be an obstacle to the use of motion disparity in the stereo-correspondence process. For instance, the studies reviewed by Le Grand (1956) suggest that the stereo correspondence based on $I$ is achieved early in the visual pathway, independently of any signal related to eye position. In addition, the role of motion disparity could be masked, for motion $O$, by a degradation of static disparity processes due to retinal image motion.

As stated above, our failure to demonstrate a role of motion disparity in the perception of 3-D structure does 
not rule out its possible use by the visual system for the detection of the rate of change of disparity and the perception of 3-D motion. In conclusion, our results did not validate the hypothesis on motion disparity (CornilleauPérès \& Droulez, 1992), but strongly support the existence of cooperative mechanisms in stereopsis and the processing of motion parallax. An adequate model of kinetic stereopsis is still lacking.

\section{REFERENCES}

BeverLey, K. I., \& Regan, D. (1973). Evidence for the existence of neural mechanisms selectively sensitive to the direction of movement in space. Journal of Physiology, 235, 17-29.

BLAKE, R., Fox, R. (1973). The psychophysical inquiry into binocular summation. Perception \& Psychophysics, 14, 161-185.

Braunstein, M. L., Andersen, G. J., Rouse, M. W., \& Tittle, J. S. (1986). Recovering viewer-centered depth from disparity, occlusion, and velocity gradients. Perception \& Psychophysics, 40, 216-224.

Braunstein, M., \& TodD, J. (1990). On the distinction between artifacts and information. Joumal of Experimental Psychology: Human Perception \& Performance, 16, 211-216.

BülthoFf, H. H., \& MALLOT, H. A. (1987). Interaction of different modules in depth perception. In Proceedings of the First International Conference on Computer Vision (pp. 295-305). Washington, DC: IEEE Computer Society Press.

Cornilleau-PÉrès, V., \& Droulez, J. (1989). Visual perception of surface curvature: Psychophysics of curvature detection induced by motion parallax. Perception \& Psychophysics, 46, 351-364.

Cornilleau-PÉrès, V., Droulez, J. (1990). Stereo-correspondence from optic flow. In O. Faugeras (Ed.), Proceedings of the First European Conference on Computer Vision (pp. 326-330). Berlin: Springer-Verlag.

Cornilleau-Pérès, V., \& Droulez, J. (in press). Velocity correspondence in stereokinetic images. Computer Vision, Graphics \& Image Processing. Image Understanding.

Cynader, M., Regan, D. (1978). Neurons in cat parastriate cortex sensitive to the direction of motion in three-dimensional space. Journal of Physiology, 274, 549-569.

Cynader, M., \& Regan, D. (1982). Neurons in cat visual cortex tuned to the direction of motion in depth: Effect of positional disparity. $\mathrm{Vi}$ sion Research, 22, 967-982.

Droulez, J., \& Cornilleau-Pérès, V. (1990). Visual perception of surface curvature: The spin variation and its physiological implications. Biological Cybernetics, 62, 211-224.

FoleY, J. M. (1978). Primary distance perception. In R. Held, H. W Leibowitz, \& H. L. Teuber (Eds.), Handbook of sensory physiology: Vol. 8. Perception (pp. 181-213). Berlin: Springer-Verlag.

GOGEL, W. C. (1980). The sensing of retinal motion. Perception \& Psychophysics, 28, 155-163.

Green, B. F. (1959). Kinetic depth effect (Psychology Group 58, Quarterly Progress Report). Cambridge, MA: Massachusetts Institute of Technology, Lincoln Laboratory.

Green, D. M., SWets, J. A. (1974). Signal detection theory and psychophysics. New York: Kreiger.

Hildreth, E. C. (1984). The computation of the velocity field. Proceedings of the Royal Society: Series B, 221, 189-220.

HoRn, B. K. P., SChunck, B. G. (1981). Determining optical flow. Artificial Intelligence, 17, 185-203.

Husain, M., Treue, S., \& Andersen, R. A. (1989). Surface interpolation in 3-D structure-from-motion perception. Neural Computation. 1, 324-333.

JENKIN, M. R. M. (1984). The stereopsis of time-varying imagery (Tech. Rep. RBCV-TR-84-3). Toronto: University of Toronto.

JuLEsZ, B. (1971). Foundations of cyclopean perception. Chicago: University of Chicago Press.

LeE, D. N. (1971). Binocular stereopsis without spatial disparity. Perception \& Psychophysics, 9(2B), 216-218.

Le Grand, Y. (1956). Optique Physiologique. T3: LEspace visuel. Paris: Editions de la Revue d'Optique.
Longuet-Higgins, H. C., \& Prazdny, K. (1980). The interpretation of a moving retinal image. Proceedings of the Royal Society: Series B, 208, 385-397.

MARR, D., \& PoGgio, T. (1979). A computational theory of human stereo vision. Proceedings of the Royal Society: Series B, 204, 301-328.

Maunsell, J. H. R., \& VAN Essen, D. C. (1983). Functional properties of neurons in middle temporal visual area of the macaque monkey: II. Binocular interactions and sensitivity to binocular disparity. Journal of Neurophysiology, 49, 1148-1167.

Mayhew, J. E. W., Frisby, J. P. (1981). Psychophysical and computational studies toward a theory of human stereopsis. Artificial Intelligence, 17, 349-385.

Mitiche, A. (1984). On combining stereopsis and kineopsis for space perception. In Proceedings of the First Conference on Al Applications (pp. 156-160). Washington, DC: IEEE Computer Society Press.

Mitiche, A. (1988). Three-dimensional space from optical flow correspondence. Computer Vision, Graphics \& Image Processing, 42, 306-317.

NaKayama, K. (1985). Biological image motion processing: A review. Vision Research, 25, 625-660.

NAWROT, M., \& BLAKE, R. (1991). The interplay between stereopsis and structure from motion. Perception \& Psychophysics, 49, 230-244.

Orban, G. A., Spileers, W., Gulyas, B., \& Bishop, P. O. (1986). Motion in depth selectivity of cortical cells revisited. Society for Neuroscience Abstracts, 12, 584.

Petersik, J. T. (1980). The effects of spatial and temporal factors on the perception of stroboscopic rotation stimulations. Perception, 9 , 271-283.

PetTigrew, J. D. (1973). Binocular neurones which signal change of disparity in area 18 of cat visual cortex. Nature, 241, 123-124.

PogGIO, G. F., \& TAlBot, W. H. (1981). Mechanisms of static and dynamic stereopsis in foveal cortex of the rhesus monkey. Journal of Physiology, 315, 469-492.

Regan, D., \& Beverley, K. I. (1973). The dissociation of sideways movements from movement in depth: Psychophysics. Vision Research, $13,2403-2415$.

RICHARDS, W. (1977). Selective stereoblindness. In H. Spekreijse \& L. H. van der Tweel (Eds.), Spatial contrast: Report of a workshop (pp. 109-115). Amsterdam: North-Holland.

RICHARDS, W. (1983). Structure from stereo and motion. (AI Memo No. 731). Cambridge, MA: Massachusetts Institute of Technology.

Richards, W., \& REgAN, D. (1973). A stereo field map with implications for disparity processing. Investigative Ophthalmology, 12, 904-909.

Rogers, B. J., \& Collett, T. S. (1989). The appearance of surfaces specified by motion parallax and binocular disparity. Quarterly Joumal of Experimental Psychology, 41A, 697-717.

Rogers, B. J., \& GrahaM, M. E. (1982). Similarities between motion parallax and stereopsis in human depth perception. Vision Research, 22, 261-270.

Rogers, B. J., \& Graham, M. E. (1985). Motion parallax and the perception of three-dimensional surfaces. In D. J. Ingle, M. Jeannerod, \& D. N. Lee (Eds.), Brain mechanisms and spatial vision (NATO Asi Series, pp. 95-111). Dordrecht: Martinus Nijhoff.

Rogers, B. J., \& HowARD, I. P. (1991). Differences in the mechanisms used to extract 3D siant from disparity and motion parallax cues. Investigative Ophthalmology \& Visual Science, 32. (Abstract No. 152-13)

SPERLing, G., Dosher, B. A., \& LANdy, M. S. (1990). How to study the kinetic depth effect experimentally. Journal of Experimental Psychology: Human Perception \& Performance, 16, 445-450.

Sperling, G., Landy, M. S., Dosher, B. A., de Perkins, M. (1989). Kinetic depth effect and identification of shape. Journal of Experimental Psychology: Human Perception \& Performance, 15, 826-840.

SteVENs, K. A., \& Brookes, A. (1988). Integrating stereopsis with monocular interpretations of planar surfaces. Vision Research, 28 , $371-386$.

Thомаs, J. P. \& Olzak, L. A. (1990). Cue summations in spatial discrimination. Vision Research, 30, 1865-1875.

Toyama, K., Komatsu, Y., Kasal, H., Fuji, K., \& Umetani, K., (1985). Responsiveness of Clare-Bishop neurons to visual cues associated with motion of a visual stimulus in three-dimensional space. Vision Research, 25, 407-414. 
Toyama, K., \& Kozasa, T. (1982). Responses of Clare-Bishop neurons to three-dimensional movement of a light stimulus. Vision Research. 22, 571-574.

Verri, A., \& Pogglo, T. (1986). Motion field and optical flow: Differences and qualitative properties (AI Memo No. 917). Cambridge, MA: Massachusetts Institute of Technology.

Waxman, A. M., \& Duncan, J. H. (1985). Binocular image flows: Steps toward stereo-motion fusion (Report CAR-TR-119). College Park: University of Maryland, Computer Vision Laboratory.

Waxman, A. M., \& Ulman, S. (1985). Surface structure and three dimensional motion from image flow kinematics. International Joumal of Robotics Research, 4(3), 72-94.

Westheimer, G., \& LEvi, D. M. (1987). Depth attraction and repulsion of disparate foveate stimuli. Vision Research, 27, 1361-1368.

Westheimer, G., \& McKeE, S. P. (1975). Stereoscopic acuity for moving retinal images. Joumal of the Optical Society of America, 68, 450-455.

ZEKI, S. M. (1974). Cells responding to changing image size and disparity in the cortex of the rhesus monkey. Journal of Physiology, 242, 827-841.

\section{APPENDIX}

\section{Curvature and Depth Sensitivities in Stereo Vision: Comparison of Our Results With Those Obtained by Rogers and Graham}

By using surfaces corrugated in depth in the vertical direction, Rogers and Graham (1985) determined the sensitivity to depth in stereo vision. It is of some interest to compare this depth sensitivity with the curvature thresholds we obtained in Condition $\mathbf{S}$ in the first group of experiments. But first, several differences between our experimental conditions and those of Rogers and Graham should be noted.

1. The curvature of the surfaces used by Rogers and Graham was always null in the horizontal direction. Therefore, these surfaces can be compared to a cylinder with a horizontal axis, rather than to a spherical patch, such as we used. This should not make a large difference, though, because the direction that plays a major role in curvature detection is likely to be orthogonal to the direction of frontal translation involved in the image transformation (Cornilleau-Pérès \& Droulez, 1989). In stereopsis, this direction is horizontal, and therefore the direction of curvature that plays a major role for curvature detection is vertical. It follows that curvature sensitivity should be comparable for a horizontal cylinder and a sphere. In particular, under identical experimental conditions, the model of spin variation predicts similar curvature thresholds for Rogers and Graham's stimuli and for ours.

2. The size of the images was $20^{\circ} \times 25^{\circ}$, and the dot density was $65 \mathrm{dots} / \mathrm{deg}^{2}$, for Rogers and Graham (we picked the values of their 1982 study, which seems quite similar to their 1985 study), whereas these parameters were $8^{\circ}$ in diameter with a density of $4 \mathrm{dots} / \mathrm{deg}^{2}$ for us.

3. Contrary to our surfaces of uniform curvature, the surfaces used by Rogers and Graham had curvatures that varied along the vertical direction. Therefore, the comparison of curvature thresholds for the two studies involved arbitrary choices that are detailed below.

The surfaces used by Rogers and Graham (1985) had a vertical depth profile, which is a DOG. With widths (distance between two troughs) of $8^{\circ}$ and $16^{\circ}$ for these DOGs, these surfaces are comparable to our stimuli, although they present a curvature that decreases from the peak to the troughs. In this case, Rogers and Graham found depth thresholds ranging between 7 and 22 arcsec. By using the calculus already proposed by Cornilleau-Pérès and Droulez $(1989$, p. 361), we have converted the curvature thresholds interpolated from Figures 3 and 4 into equivalent disparity thresholds. The values of these thresholds range between 24 and 122 arcsec, and they are higher than the values deduced from Rogers and Graham's results (see our Figure 11). This may be due to the difference in the display conditions that have been mentioned above. Also, Rogers and Graham seem to have used skilled subjects (essentially experimenters), and their results are in better agreement with the threshold that we found for our skilled subject (V.C.-P.), who exhibited the highest curvature sensitivity (24 arcsec).

(Manuscript received May 26, 1992 ; revision accepted for publication January 20, 1993.) 